\title{
Neutrophil Extracellular Traps Augmented Alveolar Macrophage Pyroptosis via AIM2 Inflammasome Activation in LPS-Induced ALI/ARDS
}

\author{
Haitao $\mathrm{Li}^{1}$ \\ $\mathrm{Yi} \mathrm{Li}^{2}$ \\ Chao Song ${ }^{2}$ \\ Yongbin $\mathrm{Hu}^{3}$ \\ Minhui Dai ${ }^{2}$ \\ Ben Liu ${ }^{4}$ \\ Pinhua $\operatorname{Pan}^{2}$ \\ 'First Department of Thoracic Medicine, \\ Hunan Cancer Hospital/The Affiliated \\ Cancer Hospital of Xiangya School of \\ Medicine, Central South University, \\ Changsha City, Hunan Province, People's \\ Republic of China; ${ }^{2}$ Department of \\ Respiratory and Critical Care Medicine, \\ National Key Clinical Specialty, Xiangya \\ Hospital, Central South University, \\ Changsha City, Hunan Province, People's \\ Republic of China; ${ }^{3}$ Department of \\ Pathology, Xiangya Hospital, Central \\ South University, Changsha City, Hunan \\ Province, People's Republic of China; \\ ${ }^{4}$ Department of Emergency, Xiangya \\ Hospital, Central South University, \\ Changsha City, Hunan Province, People's \\ Republic of China
}

\begin{abstract}
Background: Uncontrollable inflammation is a critical feature of gram-negative bacterial pneumonia-induced acute respiratory distress syndrome (ARDS). Both neutrophils and alveolar macrophages participate in inflammation, but how their interaction augments inflammation and triggers ARDS is unclear. The authors hypothesize that neutrophil extracellular traps (NETs), which are formed during neutrophil NETosis, partly cause alveolar macrophage pyroptosis and worsen the severity of ARDS.
\end{abstract}

Methods: The authors first analysed whether NETs and caspase-1 are involved in clinical cases of ARDS. Then, the authors employed a lipopolysaccharide (LPS)-induced ARDS model to investigate whether targeting NETs or alveolar macrophages is protective. The AIM2 sensor can bind to DNA to promote AIM2 inflammasome activation, so the authors studied whether degradation of NET DNA or silencing of the AIM2 gene could protect alveolar macrophages from pyroptosis in vitro.

Results: Analysis of aspirate supernatants from ARDS patients showed that NET and caspase-1 levels were correlated with the severity of ARDS and that the levels of NETs and caspase-1 were higher in nonsurvivors than in survivors. In vivo, the NET level and proportion of pyroptotic alveolar macrophages in bronchoalveolar lavage fluid (BALF) were obviously higher in LPS-challenged mice than in control mice $24 \mathrm{~h}$ after injury. Administration of DNase I (a NET DNA-degrading agent) and BB-Cl-amidine (a NET formation inhibitor) alleviated alveolar macrophage pyroptosis, and Ac-YVAD-cmk (a pyroptosis inhibitor) attenuated NET levels in BALF and neutrophil infiltration in alveoli. All treatments markedly attenuated the severity of ARDS. Notably, LPS causes NETs to induce alveolar macrophage pyroptosis, and degradation of NET DNA or silencing of the AIM2 gene protected against alveolar macrophage pyroptosis.

Conclusion: These findings shed light on the proinflammatory role of NETs in mediating the neutrophil-alveolar macrophage interaction, which influences the progression of ARDS.

Keywords: ALI/ARDS, NETs, macrophage pyroptosis, AIM2 inflammation

\section{Introduction}

Acute lung injury/acute respiratory distress syndrome (ALI/ARDS) is a life-threatening form of acute respiratory failure characterized by uncontrolled, overwhelming inflammatory lung injury. Diffuse alveoli and microvasculature damage resulting from inflammation result in oedema and gas exchange restriction following hypoxemia. ${ }^{1}$ Approximately $10 \%$ of patients admitted to the intensive care unit (ICU) are diagnosed with ARDS, and $23 \%$ of those patients receive mechanical
Correspondence: Pinhua Pan Department of Respiratory and Critical Care Medicine, National Key Clinical Specialty, Xiangya Hospital, Central South University, Changsha City, Hunan Province, People's Republic of China Email pinhuapan668@csu.edu.cn 
ventilation; however, the mortality rate is as high as 35$46 \% .^{2}$ To date, the application of lung-protective ventilation remains the only proven method to decrease mortality from ARDS. ${ }^{3}$ Considering that there are few available therapies, elucidating the underlying mechanisms of ALI/ ARDS will be key to developing treatments in the future.

Alveolar macrophages are the predominant immune cells in airways and maintain a homeostatic environment. ${ }^{4}$ However, macrophages may undergo pyroptosis, which is accompanied by a robust inflammatory response, in certain situations. Canonical pyroptosis is a form of caspase-1-dependent cell death with some characteristics of apoptosis (nuclease-mediated cleavage of DNA) and necrosis (cell swelling and proinflammatory intracellular content release). ${ }^{5}$ During pyroptosis, a sizeable supramolecular complex termed the inflammasome is formed. ${ }^{6}$ The inflammasome consists of a sensor protein, an adaptor protein (apoptosis-associated specklike protein containing a CARD, ASC) and procaspase-1. The main difference between canonical caspase-1 inflammasomes, including the NLRP1, AIM2, NLRP3, and NLRC4 inflammasomes, is the receptor protein. ${ }^{7}$ Following inflammasome activation, pro-caspase- 1 is cleaved to generate active caspase-1, leading to the cleavage of pro-IL-1 $\beta$ and pro-IL-18 to the mature and secreted forms IL-1 $\beta$ and IL-18, respectively. Previous studies have reported that alveolar macrophage pyroptosis occurs in a mouse model of lipopolysaccharide (LPS)induced ALI. ${ }^{8,9}$ A large number of studies have demonstrated that the inflammasome is central to IL- $1 \beta$ production in ARDS, and macrophages are thought to be the predominant sources of IL-1 $\beta$ and IL-18. ${ }^{10-12}$ Many studies have described the essential role of pyroptosis in host defence, ${ }^{13,14}$ but excessive pyroptosis can also lead to a strong inflammatory response and play a pathological role. ${ }^{8,9}$ However, the factors that affect alveolar macrophage pyroptosis in such a situation remain poorly defined.

Neutrophils are the first leukocytes recruited during inflammation, and neutrophil influx into alveoli has been identified as a feature of ALI/ARDS. ${ }^{15}$ Neutrophils eliminate invading microbes through an array of strategies, including phagocytosis, degranulation, and neutrophil extracellular traps (NETs). ${ }^{16}$ Upon activation by various stimuli, neutrophils release a network of extracellular fibril matrices, primarily consisting of DNA and histones embedded with proteases, including neutrophil elastase (NE) and myeloperoxidase (MPO), known as NETs. ${ }^{17-21}$ This process begins with reactive oxygen species (ROS) and results in protein-arginine deiminase 4 (PAD4) activation, the conversion of arginine to citrulline on histones, and subsequent chromatin decondensation in the neutrophil nucleus. ${ }^{16}$ NETs were identified as potential mediators of systemic inflammatory diseases, including sepsis and aspiration- or LPS-induced ALI. ${ }^{22-25}$ These findings indicate that NETs could aggravate alveolar-capillary barrier damage and cytokine production. Generally, NETs are removed by macrophages via phagocytosis. ${ }^{26}$ However, accumulated evidence suggests that NETs induce cytokine production in macrophages, ${ }^{27-30}$ raising the possibility that neutrophils are involved in the regulation of alveolar macrophage function through NET release.

\section{Materials and Methods}

\section{Human Subjects}

All human studies were approved by the Ethics Committee and Institutional Review Board of Xiangya Hospital, Changsha, the People's Republic of China, and performed according to the recommendations of the Declaration of Helsinki for biomedical research involving human subjects. All subjects provided informed consent before being enrolled in the study. Twenty-three ARDS patients with gram-negative bacterial pneumonia admitted to the respiratory intensive care unit (RICU) of Xiangya Hospital, Central South University, were enrolled in this study. All subjects had ARDS according to the current Berlin definition: (1) acute onset of dyspnoea with a history of aspiration within one week and incubation for mechanical ventilation support, (2) partial pressure of oxygen $\left(\mathrm{PaO}_{2}\right) / \mathrm{FiO}_{2}$ equal to or less than $300 \mathrm{mmHg}$ over $48 \mathrm{~h}$, and (3) new infiltration shadow on chest imaging. Patients who had sepsis or Staphylococcus aureus or streptococci in deep sputum were not included. Eleven age- and sex-matched healthy volunteers were enrolled as controls. Bronchoscopy was performed for all subjects diagnosed within 24 hours to obtain bronchial aspirates. Additional bronchoalveolar lavage (BAL) was also performed for healthy volunteers to obtain alveolar macrophages for the in vitro study. For routine bronchoscopy, a bronchoscope was wedged in the right middle lobe bronchus for suction to obtain aspirate. Resident human alveolar macrophages were obtained by an additional BAL of the left lung with five aliquots of $40 \mathrm{~mL}$ sterile saline. Given that the incidence of bacterial pneumonia-induced ARDS is relatively low and that this study was a preliminary evaluation rather 
than a randomized, controlled study in human patients, sample size calculation was not conducted.

\section{Animals}

Six- to eight-week-old male C57BL/6J mice weighing 18 $20 \mathrm{~g}$ were purchased from the Department of Animal Laboratory of Central South University. The mice were raised in an air-conditioned environment at $25^{\circ} \mathrm{C}$ on a 12 $\mathrm{h}$ dark-light cycle and allowed to acclimate for three days upon arrival before experimentation. The experimental protocols performed in our study followed the Regulations for the Administration of Affairs Concerning Experimental Animals and were reviewed and approved by the Experimental Animal Ethics Committee of the Department of Animal Laboratory, Central South University, Changsha, People's Republic of China.

\section{Induction of Lung Injury in Mice by Tracheal LPS Administration and Treatment with DNase I, BB-Cl-Amidine, Ac-YVAD-cmk}

To induce LPS-induced ALI, mice were anaesthetized using a mixture of ketamine $(87.5 \mathrm{mg} / \mathrm{kg})$ and xylazine $(12.5 \mathrm{mg} / \mathrm{kg})$. Orotracheal intubation was performed with a 20-gauge intravenous catheter, and LPS (Sigma-Aldrich, MO, USA) was instilled at a dose of $4 \mathrm{~mL} / \mathrm{kg}$. Phosphatebuffered saline (PBS) served as control. After LPS or PBS installation, to allow the instilled solution to distribute throughout the lungs, the mice were mechanically ventilated with a rodent ventilator (VentElite, Harvard Apparatus, MA, USA) using room air and a volume-controlled setting as described previously. ${ }^{31}$ The following parameters were used: $7 \mathrm{~mL} / \mathrm{kg}$ body weight, 120 breaths/min, and a positive end-expiratory pressure (PEEP) of $2 \mathrm{~cm} \mathrm{H}_{2} \mathrm{O}$ for 10 minutes. After the mice were weaned off the ventilator, they were monitored in the animal care facility for the subsequent $24 \mathrm{~h}$.

In a separate set of experiments, we sought to determine whether the combination of neutrophil NETosis and macrophage pyroptosis contributes to the pathogenesis of ARDS. To this end, DNase I (5 mg/kg; Roche, Basel, Switzerland) instilled into the lungs of some mice shortly after LPS challenge, while other mice received BB-Clamidine (1 mg/kg; Cayman Chemical, MI, USA) or AcYVAD-cmk (6.5 mg/kg; Sigma-Aldrich, MO, USA) intraperitoneally. The dosages of DNase I, BB-Cl-amidine, and Ac-YVAD-cmk were selected according to previous experiments. ${ }^{8,25,32}$ All mice were sacrificed $24 \mathrm{~h}$ later.

\section{Preparation of Clinical and Mouse Samples}

Human aspirate smears were prepared for qualitative analysis of NETs by immunofluorescence and cytological examination by haematoxylin-eosin (HE) staining. The aspirates were homogenized with $0.1 \%$ dithiothreitol (DTT) and centrifuged at $1000 \times \mathrm{g}$ at $4^{\circ} \mathrm{C}$, and the cell-free supernatants were stored at $-80^{\circ} \mathrm{C}$ for further quantification of NET, caspase-1, and inflammatory indicator levels.

After the mice were sacrificed, the lungs were flushed with $1 \mathrm{~mL}$ PBS to obtain bronchoalveolar lavage fluid (BALF). BALF was centrifuged at $400 \times \mathrm{g}$ to collect precipitated cells for leukocyte classification and analysis of alveolar macrophage pyroptosis, while BALF supernatants were stored at $-80^{\circ} \mathrm{C}$ for further quantification of NET, protein and local inflammatory indicator levels. The lung lobes were excised and cut into three parts: one part was weighed to calculate the dry-wet weight ratio, one part was ground into a homogenate and stored at $-80^{\circ} \mathrm{C}$ for Western blotting, and the last part was fixed with $4 \%$ buffered paraformaldehyde and subjected to HE staining and immunofluorescence. Mouse blood was collected in a tube containing heparin as an anticoagulant by left ventricular puncture; an aliquot of blood was sent for blood-gas analysis, and the rest of the blood was centrifuged to isolate plasma for quantification of systemic inflammatory indicator levels.

\section{Assessment of Lung Injury in Mice}

For blood-gas analysis, the arterial $\mathrm{PaO}_{2}$ of anticoagulated arterial blood from the left ventricle was analysed with a gas analyser within half an hour.

To determine the ratio of wet/dry weight of the lungs, the left lower lobes were quickly rinsed in PBS to remove residual blood. The wet weight of the lung lobes was determined after the excess PBS was carefully removed with tissue paper. Then, the lobes were placed in an oven at $58^{\circ} \mathrm{C}$ for $48 \mathrm{~h}$ and weight again to obtain the dry weight. The dry/wet ratio of the mouse lung tissues was calculated to evaluate the degree of pulmonary oedema.

For lung histology, after being fixed with $4 \%$ buffered paraformaldehyde for $48 \mathrm{~h}$, the right lobes were dehydrated and embedded in paraffin. The paraffin tissue blocks were cut into $5-\mu \mathrm{m}$ sections, and the sections 
were mounted on glass slides, dewaxed and stained with haematoxylin and eosin. The stained sections were evaluated by pathologists blinded to the experimental design, and then subjected to standard histopathological analysis as described previously. ${ }^{25}$ Briefly, the severity of lung injury was quantitatively scored according to four indicators: haemorrhage, alveolar oedema, thickening of the alveolar septa and leukocyte infiltration. Each indicator was assigned a score ranging from $0-3$ according to severity $(0=$ normal; $1=$ mild; $2=$ moderate; $3=$ severe $)$. The indicator scores were summed to obtain the pathological lung injury score.

To measure protein levels in BALF, the protein concentration in mouse BALF supernatant was determined by the bicinchoninic acid protein assay (Biomiga, USA).

To classify leukocytes in BALF, cells precipitated from mouse BALF obtained as described above were resuspended in medium, seeded on 15-mm glass slides in 24well plates and kept at $37^{\circ} \mathrm{C}$ for $1 \mathrm{~h}$ to allow the cells to adhere to the slides. The slides were air-dried and subjected to Wright's-Giemsa staining following the manufacturer's instructions. The slides were examined for to determine the numbers of different types of leukocytes.

\section{MPO-DNA Complex Enzyme-Linked Immunosorbent Assay (ELISA)}

NETs in human aspirate supernatants and mouse BALF were quantified by a method involving measurement of the MPODNA complex level as previously described. ${ }^{33,34}$ In brief, a 96-well flat-bottom plate was pre-coated with $5 \mu \mathrm{g} / \mathrm{mL}$ anti-MPO monoclonal antibody (Merck Millipore, MA, USA) as a capture antibody (diluted 1:500 in 50 $\mu \mathrm{L}$ ). After three washes (300 $\mu \mathrm{L}$ each), the cells were blocked with $1 \%$ bovine serum albumin (BSA) for $1 \mathrm{~h}$ at room temperature (RT). Samples (10 $\mu \mathrm{L} /$ well) were added to $40 \mu \mathrm{L}$ incubation buffer containing a peroxidase-labelled anti-DNA monoclonal antibody (Cell Death ELISA ${ }^{\text {PLUS }}$, component 2, diluted 1:25; Roche, Basel, Switzerland). The plate was incubated for $2 \mathrm{~h}$ at RT. After three washes $(300 \mu \mathrm{L}$ each), $50 \mu \mathrm{L}$ peroxidase substrate (ABTS) was added, and the plate was incubated for 20 minutes at RT. Then, the absorbance at 450 $\mathrm{nm}$ was measured in the dark.

\section{Visualization and Evaluation of Alveolar Macrophage Pyroptosis in Mice}

Typical pyroptosis is a caspase-1-dependent form of cell death involving nuclear DNA fragmentation. To visualize alveolar macrophage pyroptosis, we isolated alveolar macrophages and further assessed active caspase-1 levels and DNA breakage. Alveolar macrophages were isolated from BALF as described in our previous study. ${ }^{35}$ In brief, BALF cells were incubated with magnetic nanoparticle-conjugated antibodies (mouse anti-Gr-1, anti-CD4, anti-CD8, and antiCD45R/B220 antibodies; BD Biosciences Pharmingen, CA, USA) to label and remove polymorphonuclear neutrophils (PMNs) and lymphocytes through the use of immunomagnetic separation system (BD Biosciences Pharmingen, CA, USA). Residual non-labelled cells (alveolar macrophages) were incubated with FITC-labelled FAM-YVAD-FMK (FAM-FLICA Caspase-1 Assay Kit; ImmunoChemistry, $\mathrm{MN}, \mathrm{USA}$ ) at $37^{\circ} \mathrm{C}$ for $1 \mathrm{~h}$. Then, the alveolar macrophages were fixed with $4 \%$ paraformaldehyde at RT for 20 minutes. After being washed 3 times, the cells were subjected to TUNEL staining (In Situ Cell Death Detection Kit, TMR red; Sigma-Aldrich, MO, USA) following the manufacturer's instructions. Fluorescent signals for active caspase-1 and TUNEL were detected by confocal microscopy. The pyroptosis rate in each group was calculated by analysing 400 adherent alveolar macrophages in random fields.

In the early stage of pyroptosis, increased permeability of the cell membrane precedes DNA damage in the nucleus. We also used flow cytometry as an alternative strategy to evaluate alveolar macrophage pyroptosis in mice. BALF cells were incubated with FITC-labelled FAM-YVADFMK (FAM-FLICA Caspase-1 Assay Kit; ImmunoChemistry, $\mathrm{MN}$, USA) at $37^{\circ} \mathrm{C}$ for $1 \mathrm{~h}$; CD45 (Biolegend, CA, USA) Siglec-F, and CD11c antibodies (BD Bioscience, CA, USA) at RT for 15 minutes; and propidium iodide (PI) at RT for 5 minutes. Stained cells were analysed with a FACSVerse BD flow cytometer (BD Biosciences, MD, USA). The raw data were analysed using FlowJo software (TreeStar Corporation, USA). CD45-, Siglec-F-, CD11c-positive cells were considered alveolar macrophages, and FITC-labelled active caspase-1- and PIpositive cells were considered early pyroptotic cells.

\section{Human Alveolar Macrophage Isolation and Culture}

BALF obtained from healthy subjects as mentioned above was immediately placed on ice and filtered through sterile gauze to remove mucus within 15 minutes. BALF samples were centrifuged at $250 \times \mathrm{g}$ for 10 minutes at $4{ }^{\circ} \mathrm{C}$ to obtain BALF cells. The pelleted cells were washed twice in PBS and resuspended in Roswell Park Memorial Institute 
medium (RPMI) 1640 medium containing 10\% FBS. Human alveolar macrophages were purified by allowing them to adhere to culture flasks for 90 minutes at $37^{\circ} \mathrm{C}$ in a $5 \% \mathrm{CO}_{2}$ atmosphere as previously reported. ${ }^{36,37}$ Trypan blue staining was performed, and the viability of the adhere cells was determined to be $>92 \%$. Wright's-Giemsa staining was performed, and the purity of alveolar macrophages was determined to be $>95 \%$. Purified alveolar macrophages were cultured in alveolar macrophage culture medium (RPMI 1640 medium supplemented with 10\% FBS, M-CSF $100 \mathrm{U} / \mathrm{mL}$ and $1 \mathrm{mM}$ sodium pyruvate) for at least $3 \mathrm{~d}$ before further experimentation.

\section{AIM2 Gene Silencing}

AIM2 was silenced using INTERFERin (Polyplus Transfection, Illkirch, France) according to the manufacturer's instructions. Briefly, human alveolar macrophages were transfected $2 \mathrm{~d}$ after purification with 2 nM AIM2specific Silencer RNAi (Cat No: 4427037; Thermo Fisher Scientific, MA, USA). After transfection, alveolar macrophages were cultured for an additional $2 \mathrm{~d}$ in alveolar macrophage culture medium. The efficiency of AIM2 silencing was measured by RT-PCR and Western blotting as described later.

\section{Human Alveolar Macrophage Stimulation}

Alveolar macrophages were plated on glass-coated culture plates, allowed to attach for $4 \mathrm{~h}$, washed with FBS-free RPMI 1640 medium, and then treated with LPS (500 ng/ mL, Escherichia coli, 0111: B4; Invitrogen, CA, USA) or purified NETs $(2 \mu \mathrm{g} / \mathrm{mL})$. Some cells were stimulated with both LPS and purified NETs, while control macrophages were incubated with FBS-free RPMI 1640 medium for the same duration. To visualize engulfment of NETs by alveolar macrophages, the cells on the glass slides were processed for immunofluorescence. Supernatants were obtained at different time points, and NET clearance was determined by measuring the content of double-stranded (ds-DNA) in the supernatants as described later. To assess alveolar macrophage pyroptosis, cell lysates, cell culture supernatants and mRNA were all obtained at the indicated times for further analysis. To evaluate AIM2 inflammasome activation, $500 \mathrm{U} / \mathrm{mL}$ DNase I (Roche, Basel, Switzerland) was incubated in NET buffer 30 minutes before intervention, and the AIM2 gene was silenced in alveolar macrophages.

\section{Quantitative Real-Time PCR Analysis}

Total RNA was extracted using an RNeasy Micro Kit (QIAGEN, Duesseldorf, Germany) following the manufacturer's instructions. The RNA was reverse transcribed with an All-in-One ${ }^{\mathrm{TM}}$ First-Strand cDNA Synthesis Kit (GeneCopoeia, MD, USA), and then DNA was amplified with All-in-One ${ }^{\mathrm{TM}}$ qPCR Mix (GeneCopoeia, MD, USA). The sequences of the primer pairs were as follows: GAPDH forward primer, 5'-CAAATGGGATGAAGCA CTGA-3', and reverse primer, 5'-CGTCAAAGGTGG AGGAGTG-3'; NLRP1 forward primer, 5'-GTAGTCC TGTTCAGGTGGGTC-3', and reverse primer, 5'-AGAG TGGCTCAGCGAGTTTC-3'; AIM2 forward primer, 5'AAAGCTGGTGAAACCCCGAA-3', and reverse primer, 5'-GGCCTTAATAACCTTTATGGTGCT-3'; NLRP3 forward primer, 5'-GCATTTCCTCTCTAGCTGTTCCT-3', and reverse primer, 5'-TTAGGCTTCGGTCCACACAG AAAG-3'; and NLRC4 forward primer, 5'- GCTGAA GGCCTGAAAAACCTG-3', and reverse primer, 5'- ACG TTCATCCTGTCGATCAGTT-3'. The relative expression level was determined by the $2^{-\Delta \Delta C t}$ method. GAPDH was used as an internal reference.

\section{Immunofluorescence}

For NET detection, clinical aspirate smears were fixed in PLP fixative (1\% paraformaldehyde, $0.075 \mathrm{M}$ L-lysine monohydrate, and $0.01 \mathrm{M}$ sodium m-periodate in 1 $\mathrm{M}$ phosphate saline), dried and coated in $15 \%$ sucrose, and mouse lungs embedded in paraffin blocks were sliced ( $8 \mu \mathrm{m})$, mounted on glass slides, dewaxed and subjected to antigen retrieval with citrate buffer. All specimens were blocked. The aspirate smears were incubated with antihistone H3 (H3; 1:400; Novus Biologicals, CO, USA) and anti-NE (1:100, R\&D Systems, MN, USA) antibodies for $1 \mathrm{~h}$ at RT, whereas mouse lung sections were incubated overnight with anti-citrullinated histone H3 (cit-H3, 1:400, Abcam, MA, USA) and anti-MPO (1:100, R\&D Systems, $\mathrm{MN}, \mathrm{USA}$ ) antibodies at $4^{\circ} \mathrm{C}$. All specimens were incubated with Alexa 488-conjugated goat anti-rabbit and $\mathrm{Cy} 3-$ conjugated goat anti-mouse secondary antibodies. The slides were mounted with DAPI.

Isolated human neutrophils were seeded on polyL-lysine-coated coverslips in 24-well plates at a density of $2 \times 10^{5}$ cells/well. After allowing the cells to attach for $1 \mathrm{~h}, 500 \mathrm{nM}$ phorbol myristate acetate (PMA) was added, and the cells were cultured for $4 \mathrm{~h}$ at $37^{\circ} \mathrm{C}$ to produce NETs. The slides were fixed with $4 \%$ paraformaldehyde. 
After being processed as described above, the samples were incubated with primary antibodies against anti-citH3 (1:400, Abcam, MA, USA) and NE (1:100, R\&D Systems, MN, USA) overnight at $4^{\circ} \mathrm{C}$.

For visualization of engulfment of NETs by alveolar macrophages and AIM2 inflammasome activation, alveolar macrophages were seeded in 24-well plates containing coverslips and allowed to attach for at least $3 \mathrm{~h}$. After stimulation, the cells were fixed with ice-cold methanol, and immunostaining was performed as described above with primary antibodies against EEA-1 (1:200, Cell Signaling Technology, MA, USA) or Lamp-1 (1:400, Cell Signaling Technology, MA, USA) and NE (1:100, R\&D Systems, MN, USA) overnight at $4^{\circ} \mathrm{C}$ to assess NET phagocytosis. The cells were incubated with primary antibodies against Lamp-1 (1:100, Santa Cruz, CA, USA), AIM2 (1:800, Abcam, MA, USA) and ASC (1:100, Santa Cruz, CA, USA) at RT for $1 \mathrm{~h}$ followed by Cy3-conjugated goat anti-rat, Alexa Fluor 488-conjugated goat anti-rabbit and CL-680-conjugated antimouse secondary antibodies to assess AIM2 inflammasome activation. DAPI-containing mounting medium was then added.

\section{Cell Cytotoxicity Assay}

Cell cytotoxicity was assessed by measuring the release of lactate dehydrogenase (LDH) into the culture supernatant. LDH release was assessed by the LDH Cytotoxicity Assay Kit (Promega, USA) according to the manufacturer's instructions and was calculated as the percentage of total LDH content.

\section{Quantification of Inflammatory Indicator Levels}

IL-1 $\beta$, IL-18, IL-6, and TNF-a levels in the supernatant of human aspirates and culture supernatants were measured by using a commercially available human IL- $1 \beta$, IL-18 ELISA kit (Liankebio; Hangzhou, China), and an IL-6 and TNF- $\alpha$ ELISA kit (Anoric Biotechnology, Tianjin, China) according to the manufacturer's instructions. A Caspase-1 ELISA kit (SAB; MD, USA) was also employed to measure caspase-1 levels in the supernatants of human aspirates.

IL-1 $\beta$, TNF- $\alpha$ and keratinocyte-derived cytokine (KC) levels in the mouse plasma and BALF were also determined using commercially available mouse IL- $1 \beta$ and TNF- $\alpha$ ELISA kits (eBioscience; CA, USA) and KC
ELISA kit (Liankebio; Hangzhou, China) according to the manufacturer's instructions.

\section{Quantification of Cell-Free DNA (cf-DNA) and cf-DNA/MPO}

\section{Concentration}

The cf-DNA concentration in mouse BALF and cell culture supernatants was measured using the Quant-iT ${ }^{\mathrm{TM}}$ PicoGreen kit (Invitrogen, MA, USA) according to the manufacturer's instructions. The concentration of cfDNA bound to MPO (cf-DNA/MPO), ie, NET DNA, in the BALF of mice with ARDS was also quantitatively assessed. ${ }^{38}$ Briefly, a 96-well flat-bottom plate was coated with the anti-MPO monoclonal antibody mentioned above to capture MPO, a component of NETs, and then the amount of DNA bound to the MPO antibody was quantified with the Quant-iT ${ }^{\mathrm{TM}}$ PicoGreen kit.

\section{Western Blot Analysis}

Cell lysates and supernatants were routinely processed as described previously. Additionally, cell lysates were prepared according to a previous detailed description ${ }^{39}$ for ASC oligomerization.

Proteins were transferred to polyvinylidene fluoride (PVDF) membranes (Merck Millipore; MA, USA) after electrophoresis. The membranes were blocked with $8 \%$ skimmed milk for $1 \mathrm{~h}$ at RT and incubated with primary antibodies, including anti-cit-H3 (1:1000, Abcam, MA, USA), anti-NE (1:1000, R\&D Systems, MN, USA), anticleaved caspase-1 (1:1000, GeneTex; CA, USA), anti-IL-1 $\beta$ (1:1000, GeneTex; CA, USA), anti-ASC (1:1000, GeneTex; CA, USA) and anti-GAPDH (1:1000, Proteintech, Wuhan, China) antibodies, overnight at $4{ }^{\circ} \mathrm{C}$. After three washes (Trisbuffered saline plus $0.05 \%$ Tween-20), the membranes were incubated with a horseradish peroxidase-conjugated goat-anti rabbit or mouse secondary antibody (SAB; MD, USA) for $1 \mathrm{~h}$ at RT on a shaker. Signals were detected with ClarityMax Western ECL Substrate (Bio-Rad, CA, USA) and quantified by ImageJ software (Rawak Software Inc., Germany).

\section{Statistical Analysis}

All values were presented as the mean $\pm \mathrm{SD}$. No data were excluded from the final analysis. Data normality was determined with Shapiro-Wilk normality test. Correlations between variables were determined using Pearson correlation analysis. Welch's correction $t$-test or unpaired two-tailed Student's $t$-test was applied to 
compare two independent groups. One-way ANOVA followed by Tukey's post hoc test was used to analyse multiple groups. To determine the difference between two groups over time, we used two-way ANOVA followed by the Bonferroni post hoc test. The number of mice in each group was based on our preliminary experiments. The sample sizes (n) are indicated in the figures. Difference was considered significant at $\mathrm{P}<0.05$. Diagrams were generated and statistical analysis was conducted using GraphPad 8.01 software (GraphPad Software, Inc., CA, USA).

\section{Results}

\section{NET and Caspase-I Levels in Aspirates are Correlated with Disease Severity in Patients with Gram-Negative Bacterial Pneumonia-Induced ARDS}

Twenty-three ARDS patients (14 men and 9 women with a mean age of $54.39 \pm 16.43$ years) with pneumonia were enrolled in the study. All patients had a history of pulmonary gram-negative bacterial infection within one week and multiple deep sputum culture results. The baseline characteristics and demographic details of each patient are provided in the (Table 1). Based on the Berlin definition, patients were categorized into three groups according to the severity of hypoxemia: mild $\left(200 \mathrm{mmHg}<\mathrm{PaO}_{2} / \mathrm{FiO}_{2}\right.$ $\leq 300 \mathrm{mmHg}, \mathrm{n}=7)$, moderate $\left(100 \mathrm{mmHg}<\mathrm{PaO}_{2} / \mathrm{FiO}_{2}\right.$ $\leq 200 \mathrm{mmHg}, \mathrm{n}=11)$, and severe $\left(\mathrm{PaO}_{2} / \mathrm{FiO}_{2} \leq 100\right.$ $\mathrm{mmHg}, \mathrm{n}=5$ ). Among these patients, 7 (5 men and 2 women) died within $28 \mathrm{~d}$ of ARDS diagnosis.

First, we identified NETs in the bronchial aspirates of ARDS patients by double immunofluorescence staining for $\mathrm{H} 3$ and NE; no NETs were detected in healthy controls (Figure 1A). Interestingly, we noticed a few alveolar macrophages in the bronchial aspirates of healthy volunteers (Figure 1B) but massive neutrophil infiltration in the bronchial aspirates of ARDS patients (Figure 1C). Then, we measured the levels of the MPO-DNA complex (as a quantitative measure of NET levels), caspase-1 (to assess pyroptosis), and inflammatory cytokines (to evaluate inflammation) in the supernatants of aspirates from these patients. The mean MPO-DNA complex and caspase-1 levels were significantly higher in ARDS patients $(2.099 \pm 0.6998$ and $216.0 \pm 111.7$, respectively) than in healthy controls $(0.2208 \pm 0.1616$ and $26.32 \pm 6.233$, respectively; both $p<0.0001)$. Moreover, MPO-DNA complex and caspase-1 levels in bronchial aspirates correlated well with the degree of ARDS (all $p<0.05$; Figure 1D and E). Notably, MPO-DNA complex and caspase-1 levels in nonsurviving patients $(2.617 \pm$ 0.6480 and $318.5 \pm 103.5$, respectively) were significantly higher than those in survivors $(1.872 \pm 0.6084$ ad $171.1 \pm 83.38$, respectively; $p=0.0149$ and $p=0.0016$, respectively; Figure $1 \mathrm{~F}$ and $\mathrm{G})$. We also observed that the levels of inflammatory cytokines, including IL-1 $\beta$, IL-6, IL-18, and TNF- $\alpha$, were markedly increased in patients with ARDS (Supplementary Figure 1). However, the levels of these cytokines except $1 \mathrm{~L}-1 \beta$ did not correlate well with the degree of ARDS. Moreover, there were significant differences in the levels of IL-1 $\beta$ (19.82 \pm 9.440 vs $34.05 \pm 11.18 ; p=0.0048)$ and IL-18 (1732 \pm 456.7 vs $1247 \pm 464.8 ; p=0.0310$ ) between survivors and nonsurviving ARDS patients, but there were no significant differences in the levels of IL-6 and TNF-a. There was a significant correlation between MPO-DNA complex levels and caspase- 1 and IL- $1 \beta$ levels in aspirates (Figure 1), suggesting that NETs play an inflammatory role in ARDS. Because pyroptosis is dependent on the caspase-1-IL-1 axis, we concluded that NETs are closely related to pyroptosis and that both NET levels and the extent of pyroptosis might reflect the severity of ARDS in clinical situations.

\section{Inhibition of Alveolar Macrophage Pyroptosis by Ac-YVAD-cmk Reduces NET Content in an LPS-Induced ALI Mouse Model}

A clinical study suggested that NETs are associated with pyroptosis and the inflammatory response in ARDS. Notably, macrophages are the predominant cells that undergo pyroptosis, and apoptosis of neutrophils tends to be delayed in the context of bacterial infection, with these instead undergoing NETosis. ${ }^{40,41}$ Thus, to investigate the interaction between alveolar macrophage pyroptosis and neutrophil NETosis, an active caspase-1 inhibitor (AcYVAD-cmk), NET-degrading agent (DNase I) and NET formation inhibitor (BB-Cl-amidine) were administered to mice immediately after LPS challenge. Then, alveolar macrophage pyroptosis and NET content were analysed in all groups.

To determine whether alveolar macrophage pyroptosis affects neutrophil-derived NETs in vivo, we performed immunofluorescence staining of lung sections to qualitatively analyse NET formation. We found that NETs (as 
A
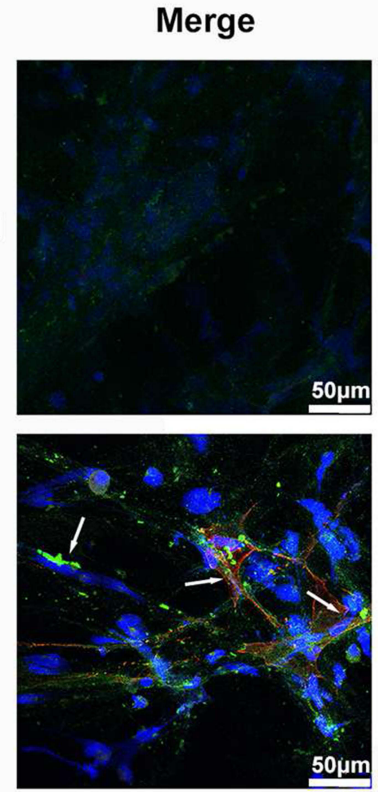

B

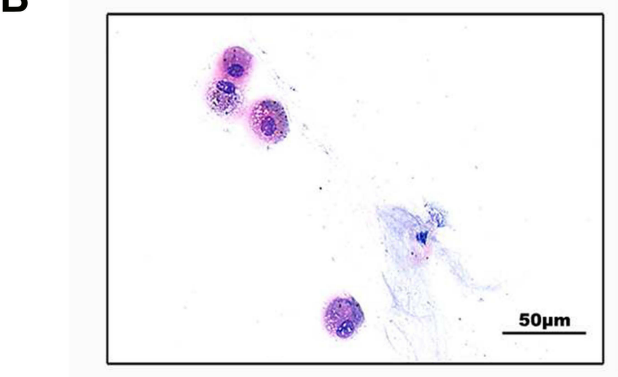

C

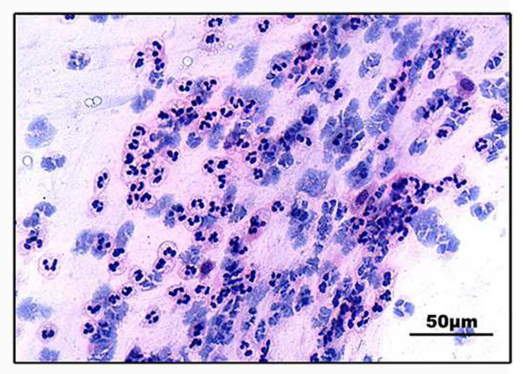

DAPI
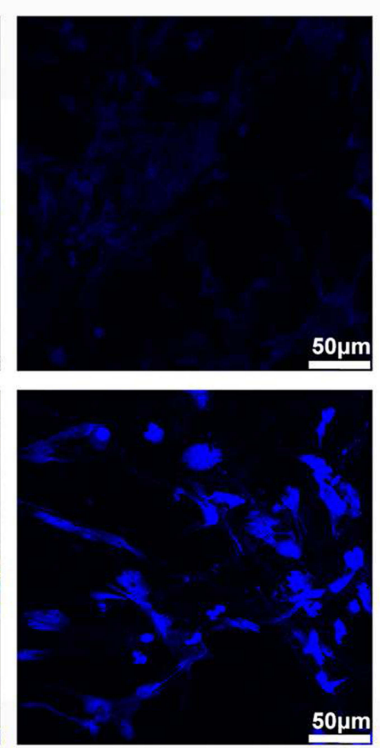

D

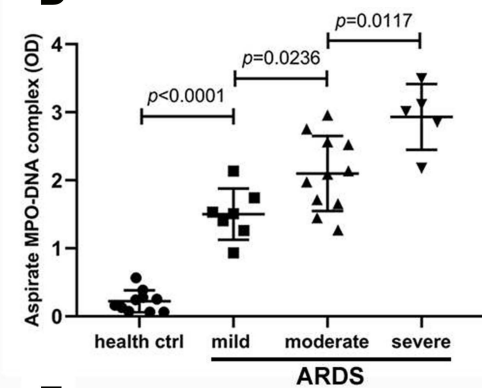

$\mathbf{F}$

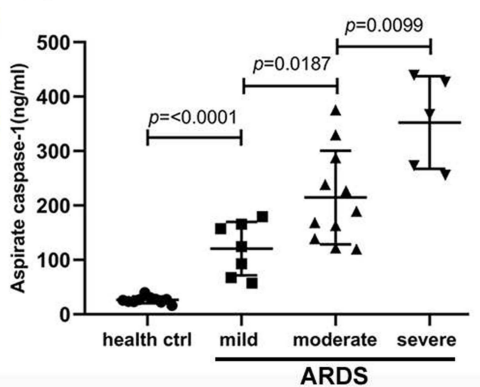

NE
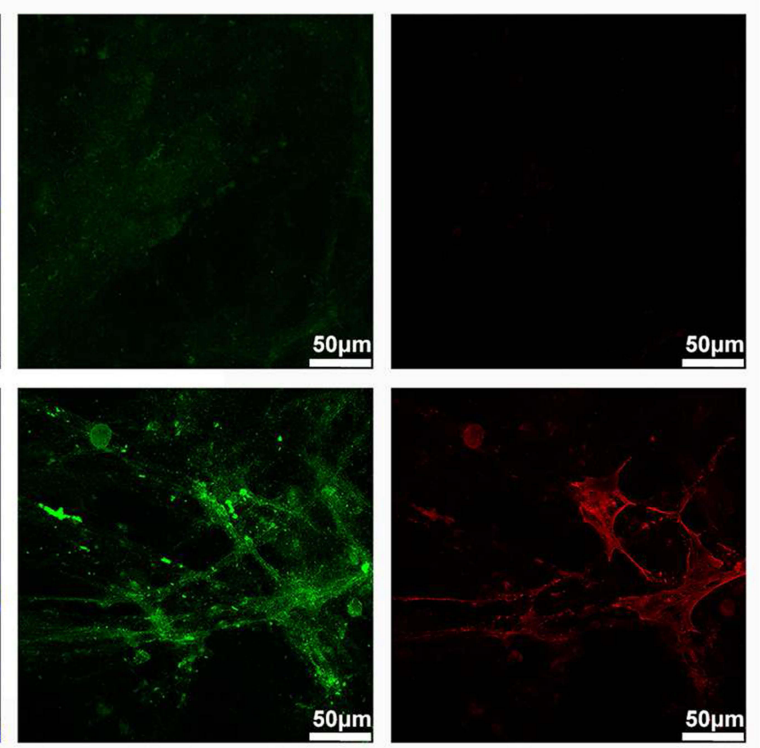

E

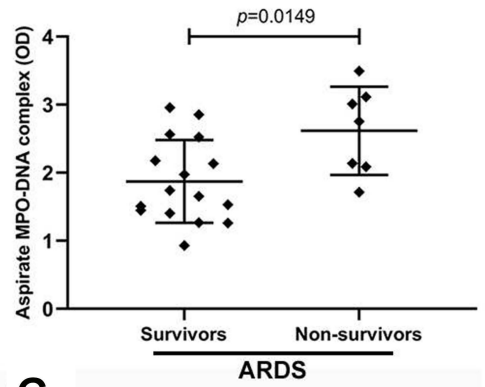

G

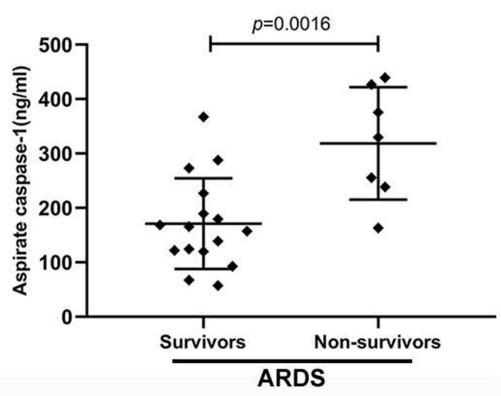

Figure I NET and caspase-I levels are increased in patients with ARDS caused by gram-negative bacteria. (A) NETs were found in the bronchial aspirates of ARDS patients but not in in healthy controls. (B and C) Alveolar macrophages were found in the bronchial aspirates of healthy volunteers, whereas massive neutrophil infiltration was found in ARDS patients. (D) NET (F) and caspase-I levels in bronchial aspirates were significantly higher in ARDS patients than in healthy controls There were also significant differences in the levels of NETs and caspase-I between patients with mild, moderate and severe ARDS. (E) NET and (G) caspase-I levels in bronchial aspirates were significantly higher in nonsurviving patients than in survivors.

Abbreviations: NETs, neutrophil extracellular traps; ARDS, acute respiratory distress syndrome.

indicated by the MPO-DNA complex) formed after LPS challenge but that NET formation was decreased in the mice that received Ac-YVAD, as well as those that received DNase I and BB-Cl-amidine (Figure 2A). As alternative approaches to quantitatively assess the role of alveolar macrophage pyroptosis in ALI-induced NET formation, Western blotting and MPO-DNA capture ELISA were employed. The results demonstrated that NET formation was attenuated in mice treated with AcYVAD-cmk compared with untreated mice after LPS challenge, as indicated by the levels of cit- $\mathrm{H} 3$ in lung tissues (Figure 2B and $\mathrm{C}$ ) and the content of the MPO-DNA 
Table I Demographics of Enrolled ARDS Patients for Sample Analysis

\begin{tabular}{|l|l|}
\hline & Patients \\
\hline Sample Sizes & 23 \\
\hline $\begin{array}{l}\text { Sex } \\
\text { Male } \\
\text { Female }\end{array}$ & 14 \\
\hline Age (years) & 9 \\
\hline Etiology & $54.39 \pm 16.43$ \\
\hline $\begin{array}{l}\text { Acinetobacter baumannii } \\
\text { Escherichia coli } \\
\text { Pseudomonas aeruginosa } \\
\text { Klebsiella pneumonia }\end{array}$ & Gram-negative bacterial \\
\hline $\begin{array}{l}\text { Category } \\
\text { Mild }(200 \mathrm{mmHg}<\mathrm{PaO} 2 / \\
\text { FiO2 } \leq 300 \mathrm{mmHg}) \\
\text { Moderate }(\mathrm{I00mmHg}<\mathrm{PaO} 2 /\end{array}$ & 13 \\
$\begin{array}{l}\text { FiO2 } \leq 200 \mathrm{mmHg}) \\
\text { Severe }(\mathrm{PaO} 2 / \mathrm{FiO} 2 \leq 100 \mathrm{mmHg})\end{array}$ & 5 \\
\hline $\begin{array}{l}\text { Outcome } \\
\text { Survival } \\
\text { Death }\end{array}$ & 11 \\
\hline
\end{tabular}

complex in BALF (Figure 2D); these findings are consistent with the results of immunofluorescence staining. Moreover, although cit-H3 expression in lung tissue was not significantly between the group treated with BB-Clamidine and the group treated with Ac-YVAD-cmk (Figure 2B and C), NET levels in the BALF of mice that received DNase I or BB-Cl-amidine were more markedly decreased than those in the BALF of mice that received Ac-YVAD-cmk (Figure 2D).

Generally, the level of NETs is also affected by neutrophil infiltration. Thus, we further assessed the effect of alveolar macrophage pyroptosis on NET production by measuring NET content and neutrophil number. Here, we calculated the ratio of NET DNA levels to the total number of neutrophils in BALF to obtain the NET index. Because both NET levels and neutrophil number were decreased in BALF, the NET index in the mice that received Ac-YVAD-cmk was comparable to that in the mice that did not receive treatment after LPS challenge (Supplementary Figure 2D). However, since DNase I or BB-Cl-amidine had little effect on neutrophil infiltration, the NET index in the mice treated with BB$\mathrm{Cl}$-amidine was reduced compared to that in LPS-challenged mice, and this decrease in the NET index was accompanied by an anti-pyroptotic effect (Supplementary Figure 2). These results indicated that alveolar macrophage pyroptosis might boost NET levels in BALF by promoting neutrophil influx into alveoli rather than directly elevating NET production in individual neutrophils.

\section{NET Degradation by DNase I or Inhibition by $\mathrm{BB}-\mathrm{Cl}-\mathrm{Amidine}$ Attenuates Alveolar Macrophage Pyroptosis in an LPS-Induced ALI Mouse Model}

Having shown that alveolar macrophage pyroptosis indirectly affects NET content in pulmonary tissue, we also attempted to determine whether NETs subsequently influence alveolar macrophage pyroptosis. As expected, tracheal injection of LPS resulted in massive neutrophil migration into alveoli and alveolar macrophage pyroptosis. The flow cytometry results showed that the percentage of pyroptotic alveolar macrophages was decreased from $24.5 \pm 2.8 \%$ in untreated mice to $17.6 \pm 1.7 \%$ or $16.1 \pm 1.6 \%$ in mice in which NETs were degraded with DNase I or NET formation was inhibited with BB-Cl-amidine (Figure 3A and D). However, there was no significant difference in the alveolar macrophage pyroptosis rate between the groups treated with this NET-targeting agents (Figure 3D). Notably, the decrease in alveolar macrophage pyroptosis in the mice treated with Ac-YVAD-cmk was even more marked, and it seemed more alveolar macrophages are survived in the mice directly inhibited pyroptosis (Figure 3C and D). Consistent with the flow cytometry results, the proportions of both active caspase-1- and TUNEL-positive alveolar macrophages in the BALF declined in ALI mice treated with DNase I $(10.81 \pm 2.60 \%)$, BB-Cl-amidine $(10.00 \pm 2.10 \%)$ or AcYVAD-CMK $(6.88 \pm 1.36 \%)$ compared to untreated ALI mice $(15.06 \pm 1.39 \%)$ (Figure 3B and E). These results indicated that NETs play a crucial role in pyroptosis of alveolar macrophages. Considering that the inhibition of NET formation and degradation of NET DNA had a similar effect on alveolar macrophage pyroptosis, NET DNA might trigger alveolar macrophage pyroptosis.

\section{NETs and Alveolar Macrophage Pyroptosis Regulate LPS-Mediated ALI and Inflammation in Mice}

We also investigated whether NETs and alveolar macrophage pyroptosis similarly affect LPS-induced severe lung injury and cytokine release. According to 


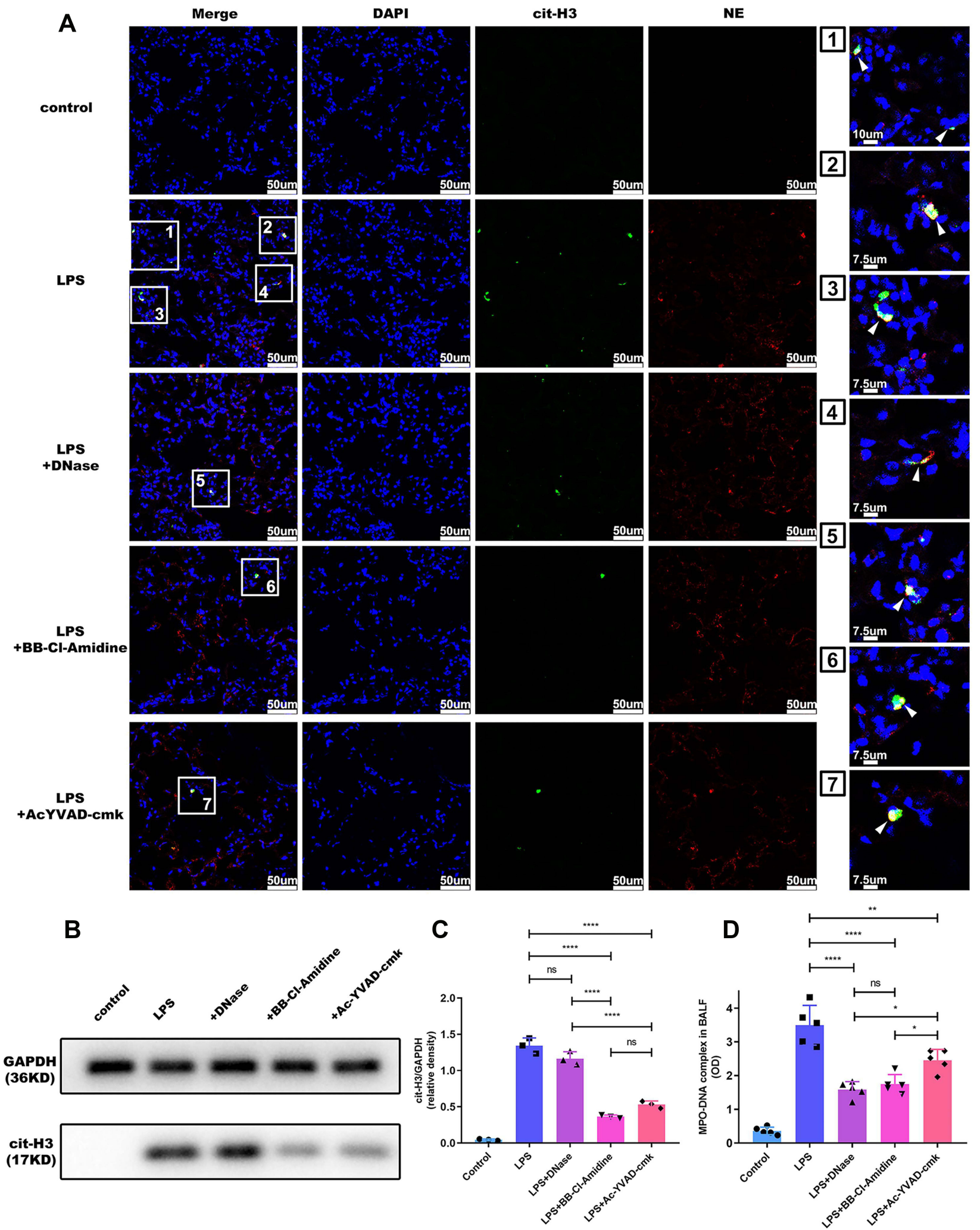

Figure 2 Alveolar macrophage pyroptosis inhibition reduces NET content in ARDS mice. Pulmonary instillation of LPS caused NET formation in mice, and NET levels were decreased in the Ac-YVAD, DNase I or BB-Cl-amidine plus LPS groups, as evidenced by immunofluorescence staining of lung sections for (A) Cit- $\mathrm{H} 3$ and $\mathrm{NE}$, (B and $\mathbf{C}$ )

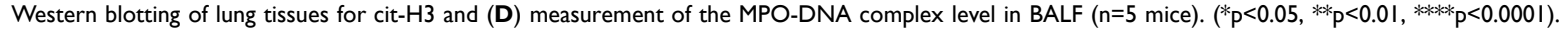

Abbreviations: NETs, neutrophil extracellular traps; ARDS, acute respiratory distress syndrome; LPS, lipopolysaccharide; cit-H3, citrullinated histone 3; NE, neutrophil elastase; MPO, Myeloperoxidase; BALF, Bronchoalveolar lavage fluid. 
A
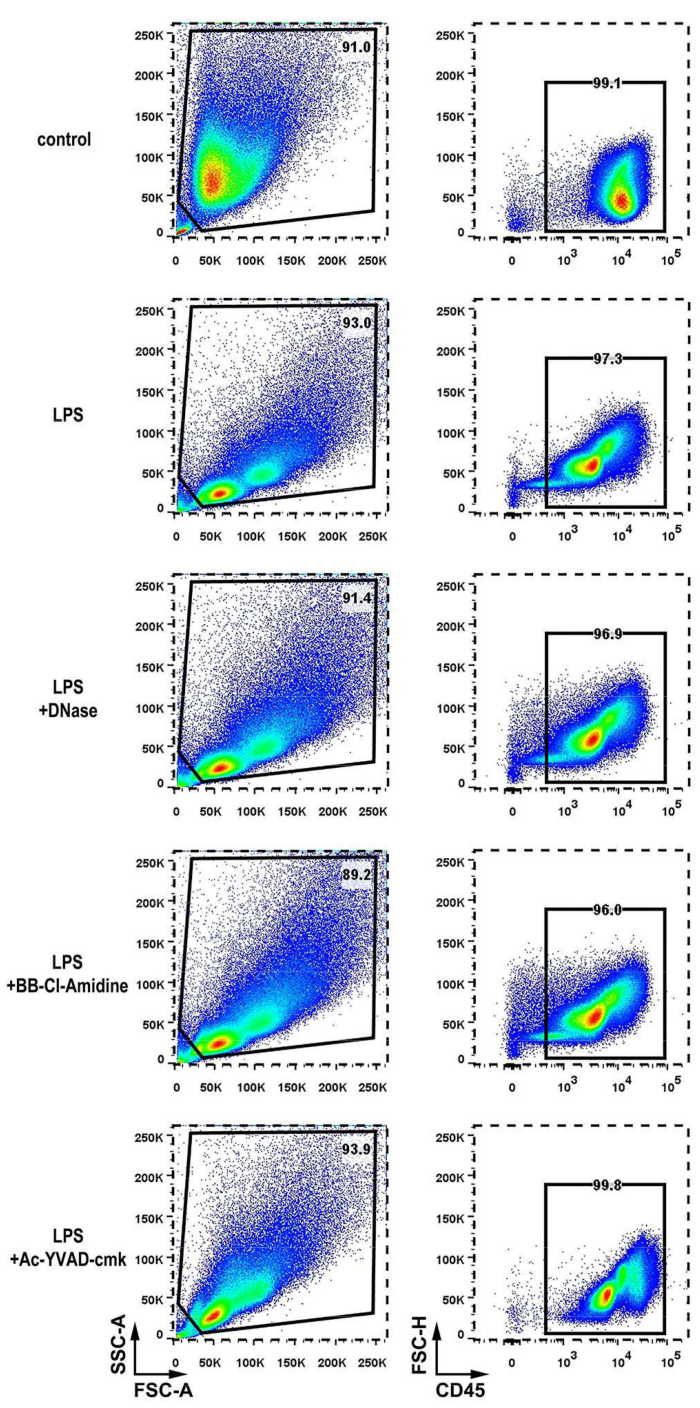

Flow cytometry
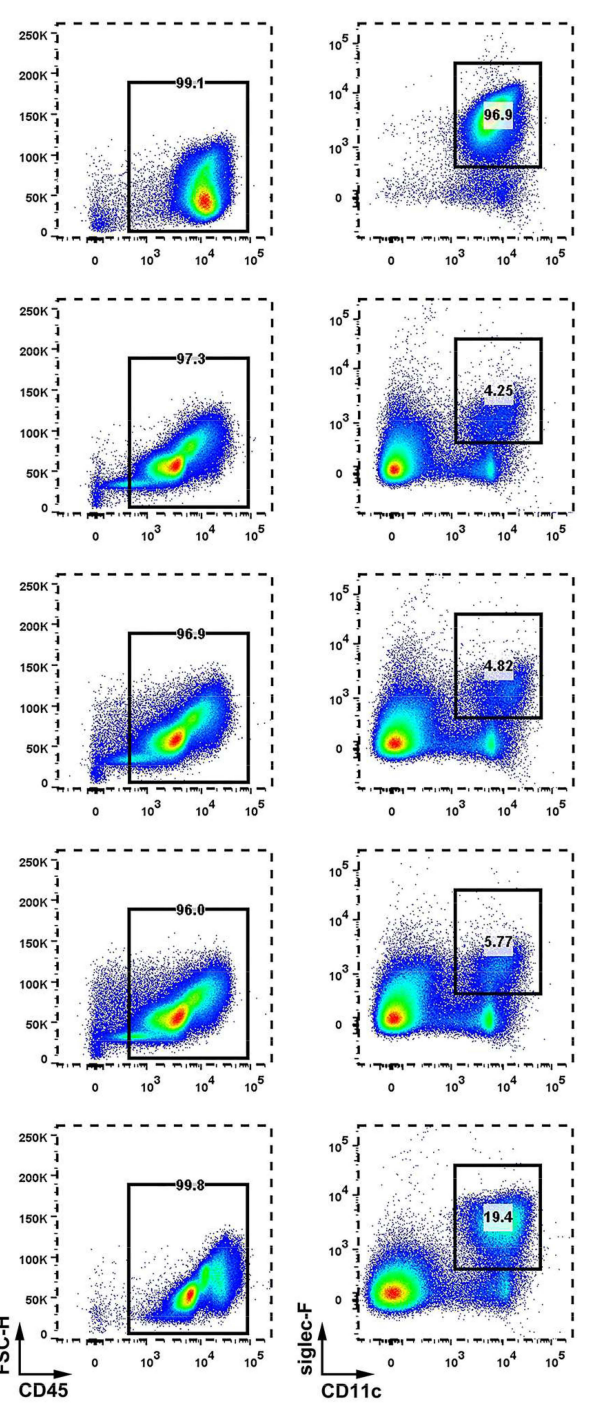

B
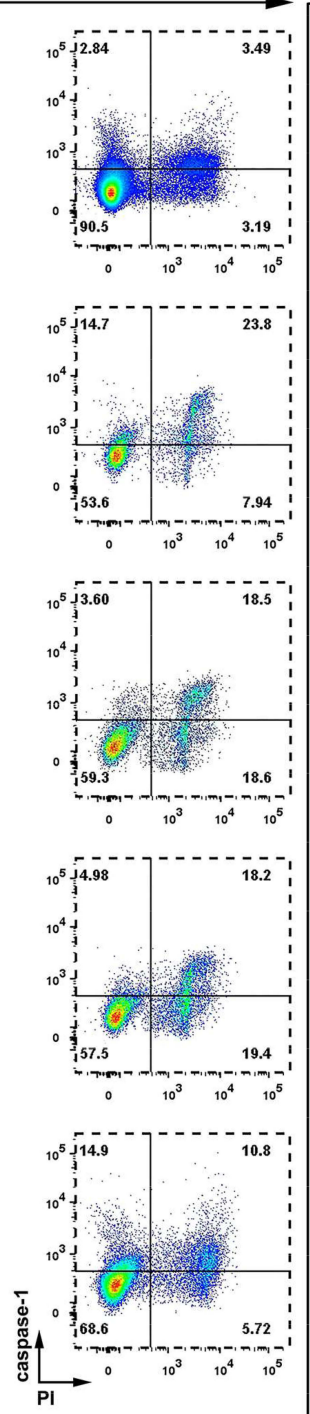

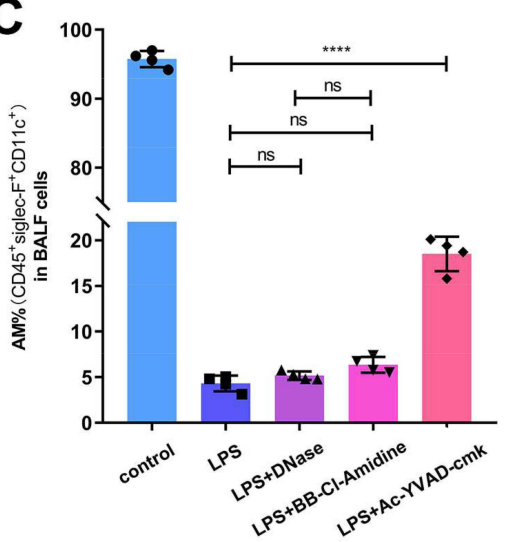

D

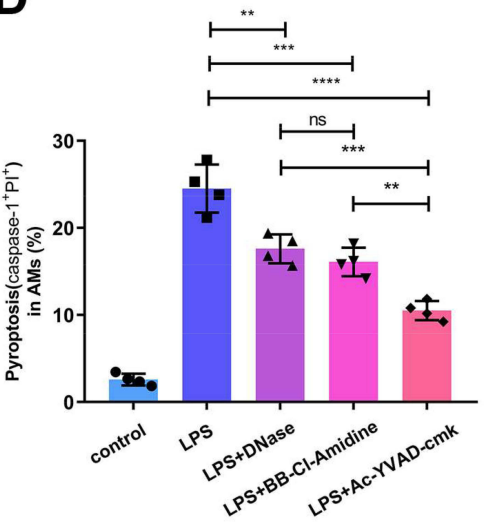

$\mathbf{E}$
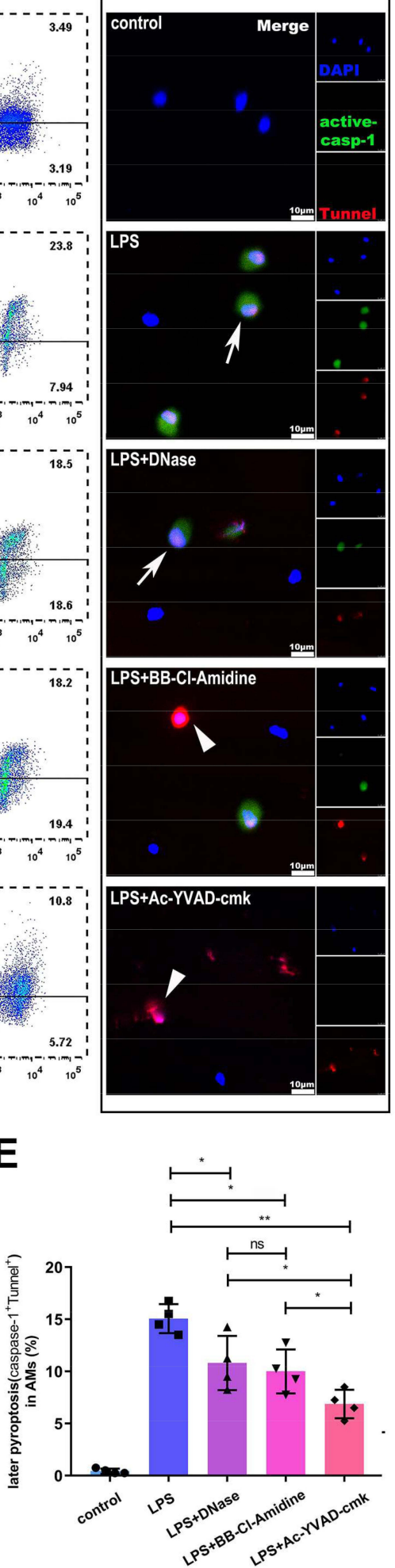

Figure 3 NET-targeting agents alleviates alveolar macrophage pyroptosis in ARDS mice. Pulmonary instillation of LPS caused alveolar macrophage pyroptosis in mice, and alveolar macrophage pyroptosis was alleviated in the Ac-YVAD, DNase I or BB-Cl-amidine plus LPS group, as evidenced by cytometry analysis of active caspase-I- and PIpositive alveolar macrophages(A and $\mathbf{C}$ )and analysis of active caspase-I- and TUNEL-positive alveolar macrophages(B and $\mathbf{E})$ in random fields by confocal microscopy. (D) The proportion of alveolar macrophages in BALF was increased in the Ac-YVAD plus LPS group compared to the LPS alone group or the DNase I and BB-Cl-amidine plus LPS group ( $\mathrm{n}=4$ mice). ( ${ }^{*} \mathrm{p}<0.05, * * \mathrm{p}<0.01, * * * \mathrm{p}<0.001$, **** $\mathrm{p}<0.0001$ ).

Abbreviations: ARDS, acute respiratory distress syndrome; LPS, lipopolysaccharide; PI, propidium iodide; TUNEL, transferase-mediated deoxyuridine triphosphate-biotin nick end labelling; BALF, bronchoalveolar lavage fluid. 
histopathological analysis, compared with no treatment, DNase I, BB-Cl-amidine and Ac-YVAD-cmk all attenuated LPS-induced haemorrhage and alveolar septal thickening after LPS challenge. Lung injury scores, the $\mathrm{PaO}_{2}$, the pulmonary wet/dry weight ratio and total protein levels were significantly reduced in the groups administered these treatments compared to the group that received LPS alone (Figure 4A-D), which indicated that both NETs and alveolar macrophage pyroptosis contribute to lung injury in mice.

To assess the impact of NETs and alveolar macrophage pyroptosis on local and systemic inflammatory responses, the levels of a panel of cytokines were quantified.

We found that the levels of interleukin-1 $\beta$ (IL-1 $\beta$ ) and tumour necrosis factor- $\alpha$ (TNF- $\alpha$ ) in BALF and serum were significantly attenuated in the groups that received a NET-targeting agent and the groups in which pyroptosis was inhibited compared to the group that received LPS alone (Figure 4E, G, H and J); additionally, the levels of $\mathrm{KC}$ in BALF was decreased in the group in which pyroptosis was inhibited (Figure $4 \mathrm{~F}$ and I). Moreover, IL-1 $\beta$ and $\mathrm{KC}$ levels in the BALF of mice in which pyroptosis was inhibited by Ac-YVAD-cmk were even lower than those in the BALF of mice in which NETs were inhibited by DNase I or BB-Cl-amidine (Figure 4E and F). In contrast, TNF- $\alpha$ levels in the BALF and serum were comparable in mice in which pyroptosis was inhibited and those in which NETs were inhibited (Figure 4G and J). Pyroptosis results in the release of proinflammatory contents from cells, especially inflammasome-activated IL-1 $\beta$; KC, an analogue of human IL-8 (CXCL-1) in mice, is mainly secreted by macrophages for neutrophil chemotaxis. The results indicated that Ac-YVAD-cmk more significantly affected LPS-induced alveolar macrophage pyroptosis and IL-1 $\beta$ secretion, which was beneficial for $\mathrm{KC}$ secretion and neutrophil chemotaxis, than the other treatments. These results are consistent with the abovementioned speculation that alveolar macrophage pyroptosis might increase NET content by recruiting neutrophils to alveoli in vivo.

\section{LPS Inhibits Alveolar}

\section{Macrophage-Mediated Phagocytosis of NETs and Triggers Pyroptosis}

Under normal physiological conditions, NETs are engulfed and degraded by macrophages via a silent process. ${ }^{26}$ However, as we showed in vivo, this process is altered during LPS-induced ALI. Thus, we hypothesized that LPS might affect the clearance of NETs by alveolar macrophages. To verify this hypothesis in vitro, we incubated human alveolar macrophages with NETs purified from PMA-stimulated human peripheral blood neutrophils treated with or without LPS. NET formation was assessed and confirmed by immunofluorescence (Supplementary Figure 3A). After incubation for $1 \mathrm{~h}$, NETs (stained with an antibody against NE) were internalized by early endosomes (stained with an antibody against EEA-1) in alveolar macrophages (Figure 5A). After incubation for $4 \mathrm{~h}$, NETs were transported to lysosomes (stained with an antibody against Lamp-1) for further degradation (Figure 5A). However, the transport of NETs to lysosomes was delayed in the presence of LPS (Figure 5A). Additionally, NET clearance by alveolar macrophages was quantified by measuring extracellular NET DNA levels in the supernatant. Our results showed that NET DNA content in the cell supernatant continuously decreased and plateaued at 12 $\mathrm{h}$ in the absence of LPS stimulation, while NET DNA content slowly decreased in absence of LPS stimulation beginning at $1 \mathrm{~h}$ (Figure 5D). Western blotting demonstrated that NE-containing NETs were phagocytosed by alveolar macrophages, and more residual NE remained in cells at $12 \mathrm{~h}$ after treatment with both LPS and NETs than after treatment with LPS or NETs alone (Figure 5B and C). These results suggested that LPS may affect NET phagocytosis and degradation by alveolar macrophages.

Since NETs accumulated in alveolar macrophages in the presence of LPS, we further determined whether LPS is involved in NET-mediated alveolar pyroptosis. To answer this question, we stimulated human alveolar macrophages with LPS $(500 \mathrm{ng} / \mathrm{mL})$ and/or NETs $(1 \mu \mathrm{g} /$ $\mathrm{mL}$ ) for $12 \mathrm{~h}$. Stimulation of human alveolar macrophages with LPS or NETs alone or in combination increased cell death, and the effect of the combination of LPS and NETs was the most potent (Figure 5G). Moreover, stimulation with LPS or NETs alone did not contribute to pyroptosome (ASC foci) formation or cleaved caspase-1 production, while combined stimulation resulted in intracellular pyroptosome (ASC foci) formation, the release of cleaved caspase-1 into the supernatant and a sharp elevation in inflammatory cytokine (IL-1 $\beta$ ) levels in the culture medium supernatant (Figure 5E-G). These results confirmed that NETs induce indirect alveolar macrophage pyroptosis when primed with LPS.

We were also interested in studying canonical inflammasome activation during alveolar macrophage pyroptosis 

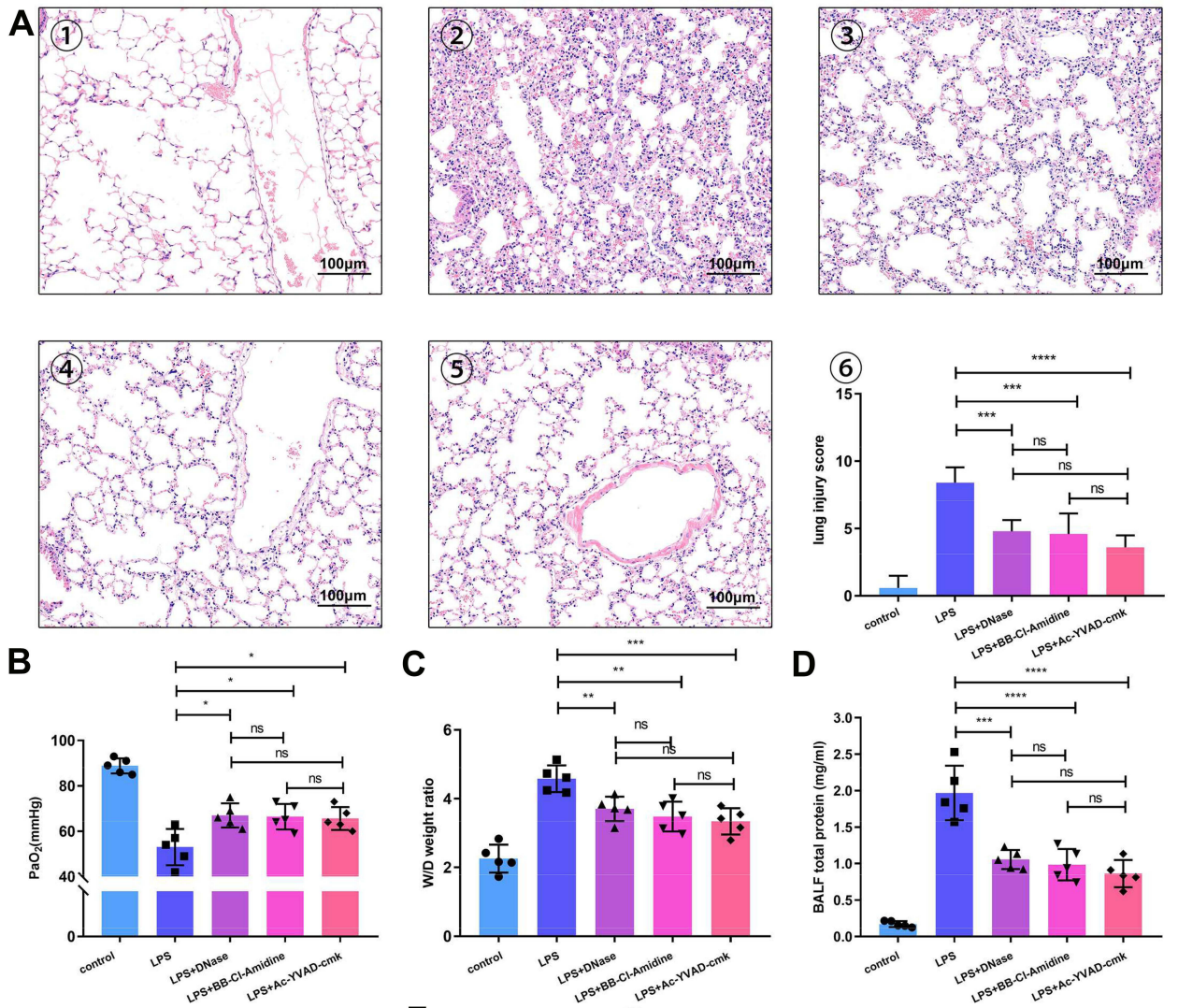

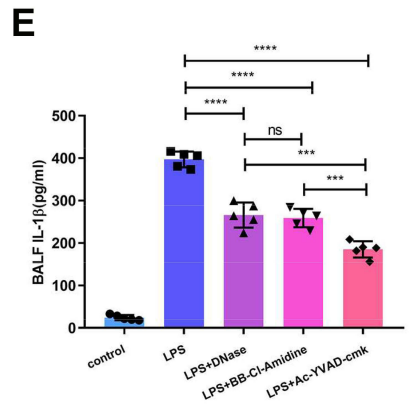

$\mathbf{F}$

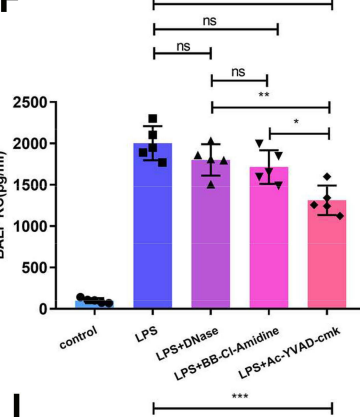

H

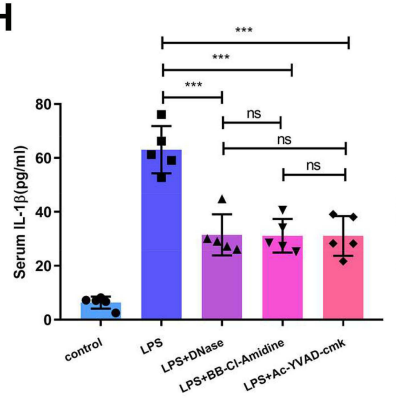

I
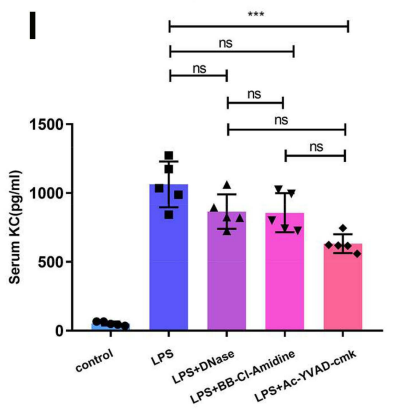

D

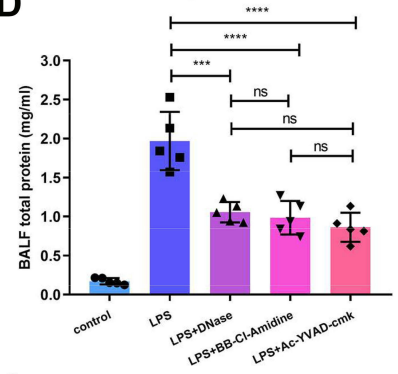

G
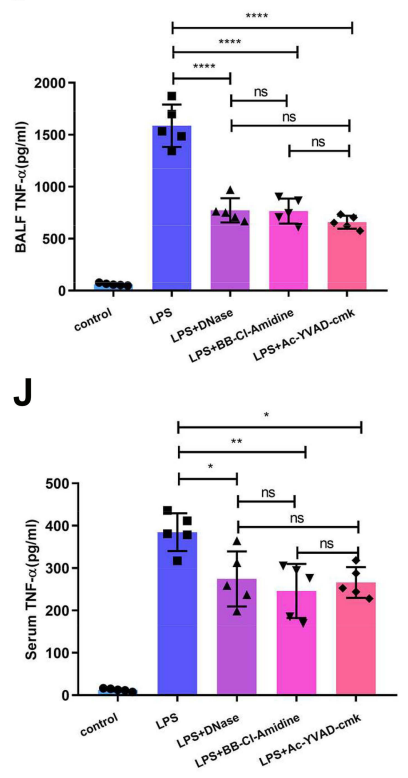

Figure 4 NET-targeting agents and inhibition of alveolar macrophage pyroptosis provide similar protection against ARDS. (A) Administration of Ac-YVAD, DNase I or BB$\mathrm{Cl}$-amidine significantly reduced lung damage in LPS-challenged mice. Representative H\&E-stained sections showing a significant improvement in the lungs of Ac-YVAD-, DNase I- or BB-Cl-amidine-treated mice. (1) Sham-treated mice, (2) LPS-treated mice, (3) DNase I plus LPS-treated mice, (4) BB-Cl-amidine plus LPS-treated mice, and (5) Ac-YVAD plus LPS-treated mice (200X). Administration of Ac-YVAD, DNase I or BB-Cl-amidine improved (B) the PaO2 of LPS-challenged mice and reduced (C) the weight/dry ratio of lung tissue and (D) the protein level in the BALF of LPS-challenged mice. BALF and serum (E and $\mathbf{H}) \mathrm{IL}-\mathrm{I} \beta,(\mathbf{F}$ and $\mathbf{I}) \mathrm{KC}$, and (G and $\mathbf{J}) \mathrm{TNF}-\mathrm{a}$ levels were all notably decreased in the DNase I, BB-Cl-amidine or Ac-YVAD plus LPS groups compared with the LPS-challenged group ( $n=5$ mice). $\left({ }^{*} p<0.05\right.$, $* * p<0.0$ I, $* * * p<0.00$ I, $* * * * p<0.0001)$.

Abbreviations: ARDS, acute respiratory distress syndrome; BALF, bronchoalveolar lavage fluid; LPS, lipopolysaccharide; IL-I $\beta$, interleukin-I $\beta$; KC, keratinocyte-derived cytokine; TNF- $\alpha$, tumor necrosis factor- $\alpha$. 
A
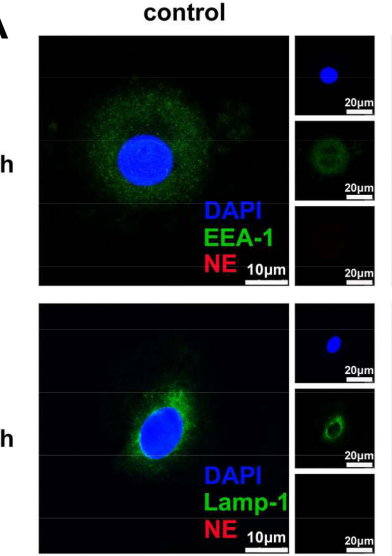

B

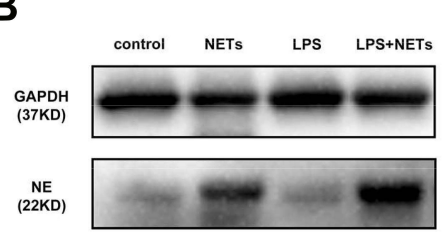

\section{E}

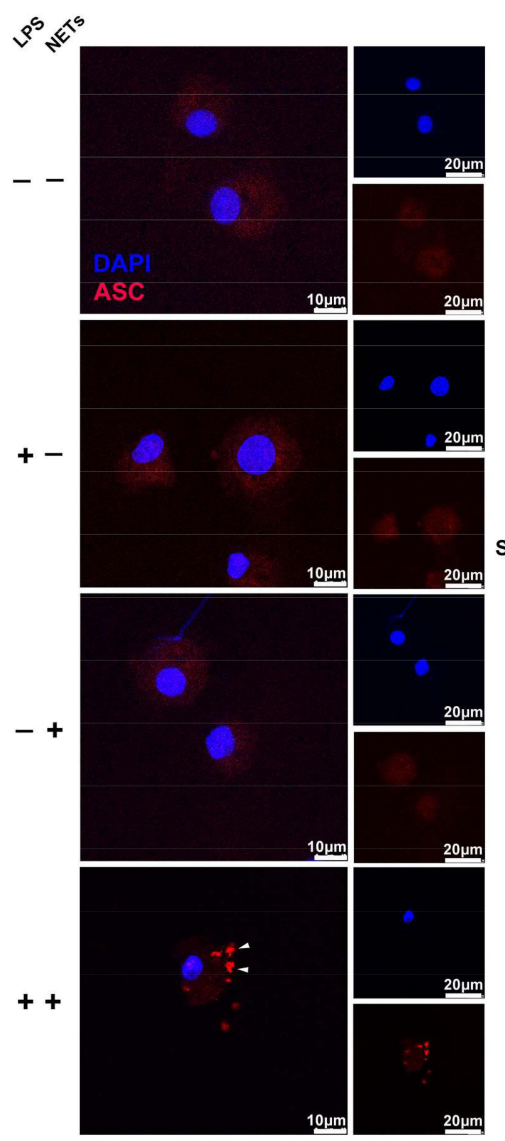

NETs
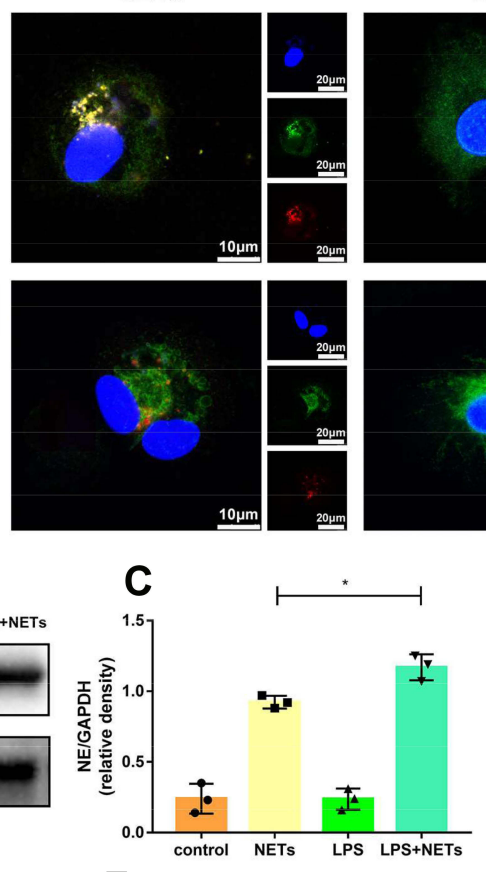

D

LPS
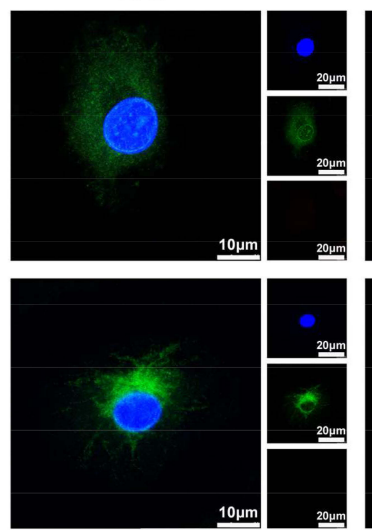

LPS+NETS
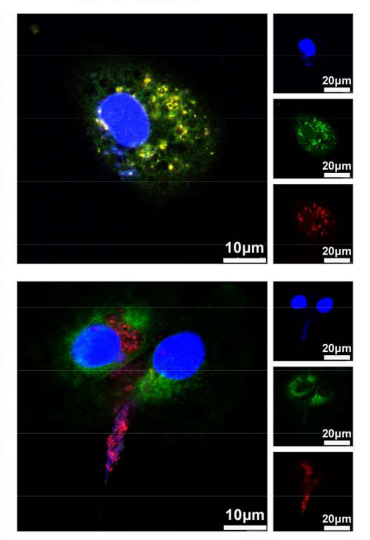

- NETs alone

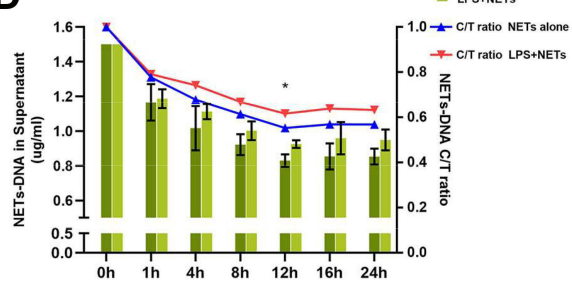

F NETS $\quad-\quad+\quad+$

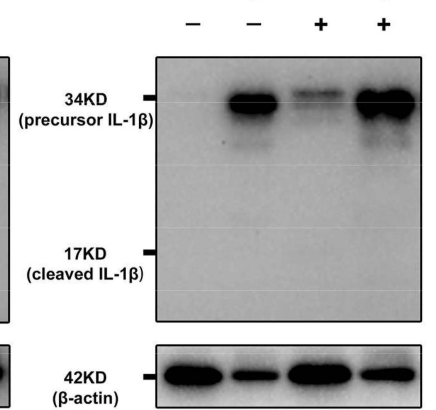

(GAPDH)

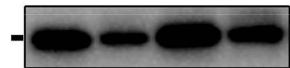

$\underset{(\beta \text {-actin })}{42 K D}$
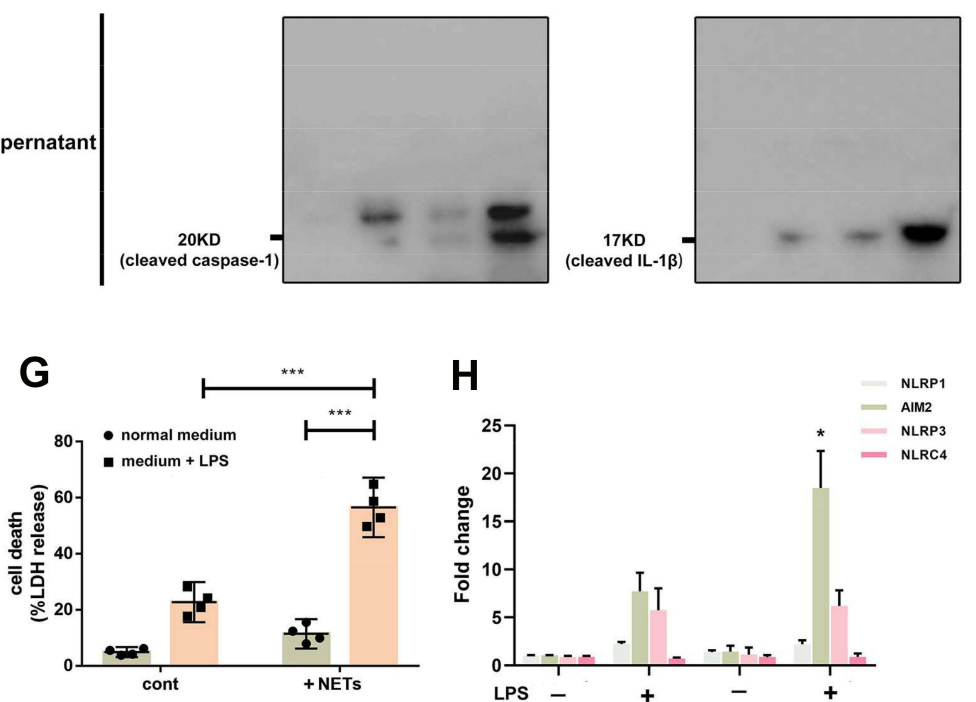

H

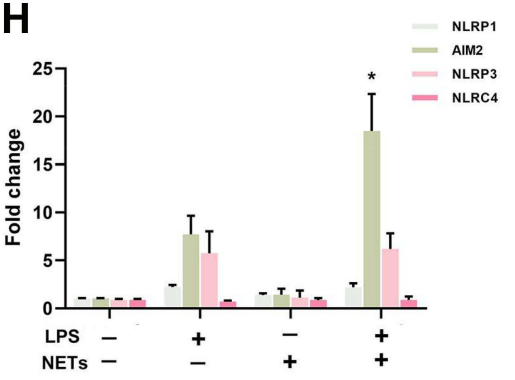

Figure 5 Delayed phagocytosis and degradation of NETs by human alveolar macrophages induces pyroptosis in the presence of LPS. (A) NETs were internalized by early endosomes in alveolar macrophages, but their transport to lysosomes was delayed. (B and C) NE accumulated in alveolar macrophages stimulated with LPS and NETs at $12 \mathrm{~h}$. (D) The decrease in NET DNA content was less marked when alveolar macrophages were coincubated with LPS and NETs than when they were treated with LPS or NETs alone, but NET DNA content reached a plateau at $12 \mathrm{~h}$. Incubation with LPS and NETs resulted in (E) intracellular pyroptosome (ASC speck) formation, (F) cleaved caspase-I expression and sharp elevation of IL-I $\beta$ levels in the supernatant, (G) significant enhancement of LDH release, and (H) a dramatic increase in AIM2 mRNA expression. ( $* p<0.05, * * * p<0.001)$. 
induced by the combination of LPS and NETs. Intracellular NLRP1 and NLRC4 mRNA expression was unaffected by any of the interventions (Figure $5 \mathrm{H}$ ). However, we observed that AIM2 and NLRP3 mRNA levels were increased in alveolar macrophages stimulated with LPS alone, while AIM2 and NLRP3 mRNA levels were unaffected by treatment with NETs alone. Unexpectedly, RT-qPCR revealed that the combination of LPS and NETs substantially increased AIM2 mRNA expression in alveolar macrophages without affecting NLRP3 mRNA expression (Figure 5H). These results suggested that NETs may mediate alveolar macrophage pyroptosis by targeting AIM2 inflammasome activation.

\section{Silencing of the AIM2 Gene or Degradation of NET DNA Alleviates Alveolar Macrophage Pyroptosis}

AIM2 is a component of the inflammasome and acts as a cytosolic ds-DNA sensor that binds ds-DNA. ds-DNA is the main constituent of NETs, and we hypothesized that phagocytized intracellular NET DNA might bind the AIM2 sensor and activate the AIM2-ASC-caspase-1 signalling pathway following alveolar macrophage pyroptosis.

AIM2 expression in alveolar macrophages was dramatically decreased after AIM2 siRNA treatment (Supplementary Figure 3B and C). Since alveolar macrophages were not vulnerable to the effects of DNase I pretreatment or AIM2 silencing (Figure 6A), alveolar macrophages were incubated with LPS and NETs, and pyroptosis was assessed. We found that alveolar macrophages underwent pyroptosis when they were incubated with LPS and NETs for $12 \mathrm{~h}$. Alveolar macrophage pyroptosis was confirmed by elevation of $\mathrm{LDH}$ release and cleaved caspase-1 production in the cell culture supernatant (Figure 6B and C). The immunofluorescence results showed that NET DNA accumulated in lysosomes bound to intracellular AIM2 receptors and caused ASC oligomerization (Figure 6D). ASC oligomerization was also confirmed by Western blotting (Figure 6E). However, alveolar macrophage pyroptosis could be alleviated by AIM2 siRNA pretreatment or rescued by the addition of DNase I (Figure 6B and C). After AIM2 gene expression was silenced, accumulated NET DNA failed to activate the AIM2 inflammasome. Degradation of NET DNA by DNase I also failed to induce ASC oligomerization (Figure 6D and E). Administration of both AIM2 siRNA and DNase I prevented the interaction between active AIM2-like receptors (ALRs) and ASC, inducing the formation of ASC specks, which recruited pro-caspase-1 to induce the autoactivation of caspase- 1 and the release of proinflammatory cytokines, such as IL-1 $\beta$, IL-6, IL-18, and TNF-a (Figure 6F-G). These results indicated that AIM2-ASC-caspase-1 signalling plays a crucial role in LPS/NET-induced alveolar macrophage pyroptosis.

\section{Discussion}

To date, ALI/ARDS is the leading cause of respiratory failure in critically ill patients and is associated with very high morbidity and mortality rates. ${ }^{1,42,43}$ Neutrophils and macrophages, the predominant inflammatory cells in alveoli, are involved in ALI/ARDS. ${ }^{44}$ The role of neutrophils and alveolar macrophages in the regulation of inflammation in ALI/ARDS is unclear, as neutrophils and macrophages are challenging to study in vivo and in vitro and can serve proinflammatory functions. However, infiltration of neutrophils and active macrophages cannot explain the overwhelming systemic inflammation that occurs in ALI/ARDS. Neutrophil-macrophage cooperation has been observed in atherosclerosis, liver injury, kidney diseases, inflammatory bowel disease and haematological diseases. ${ }^{45-48}$ Therefore, during ARDS, resident alveolar macrophages and infiltrated neutrophils may also interact to some degree. However, there has been little research on the neutrophil-alveolar macrophage interaction and its role in the uncontrolled silencing inflammation that occurs in LPS-induced ARDS.

Based on the results of our previous study, neutrophilderived NETs and caspase-1-dependent alveolar macrophage pyroptosis both contribute to inflammation in ARDS. ${ }^{9,24,49}$ Here, we investigated the roles of NETs and caspase-1 in bronchial aspirates from gram-negative bacteria-induced ARDS patients for the first time. We found statistically significant differences in NET and caspase-1 levels I the bronchial aspirate supernatants of patients with ARDS of varying severity and prognosis: the more severely ill the patients were and the higher the mortality rate was, the higher the NET and caspase-1 levels in the bronchial aspirates. These results indicated that there is a strong correlation between NET content and caspase- 1 levels in ARDS patients. As caspase- 1 is mainly released from pyroptotic alveolar macrophages, we speculate that there is an association between increased neutrophil-derived NET levels and augmented alveolar macrophage pyroptosis in the development of ARDS. 
A
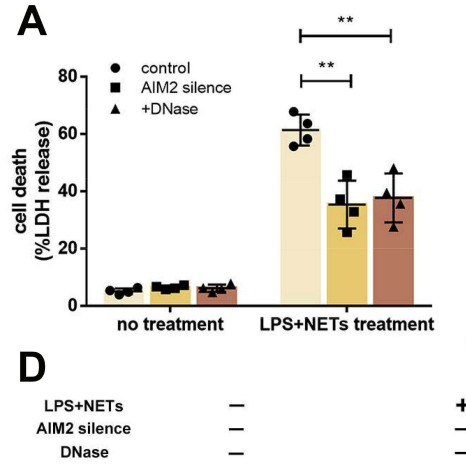

DNase

Merge

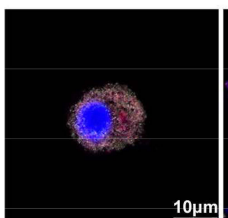

B

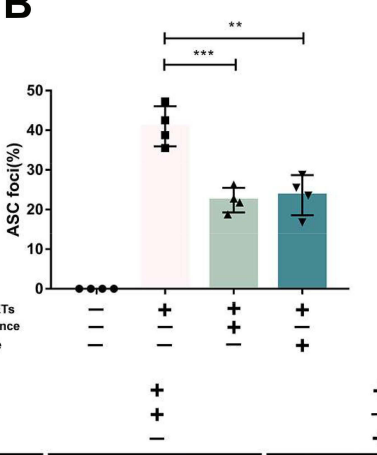

C
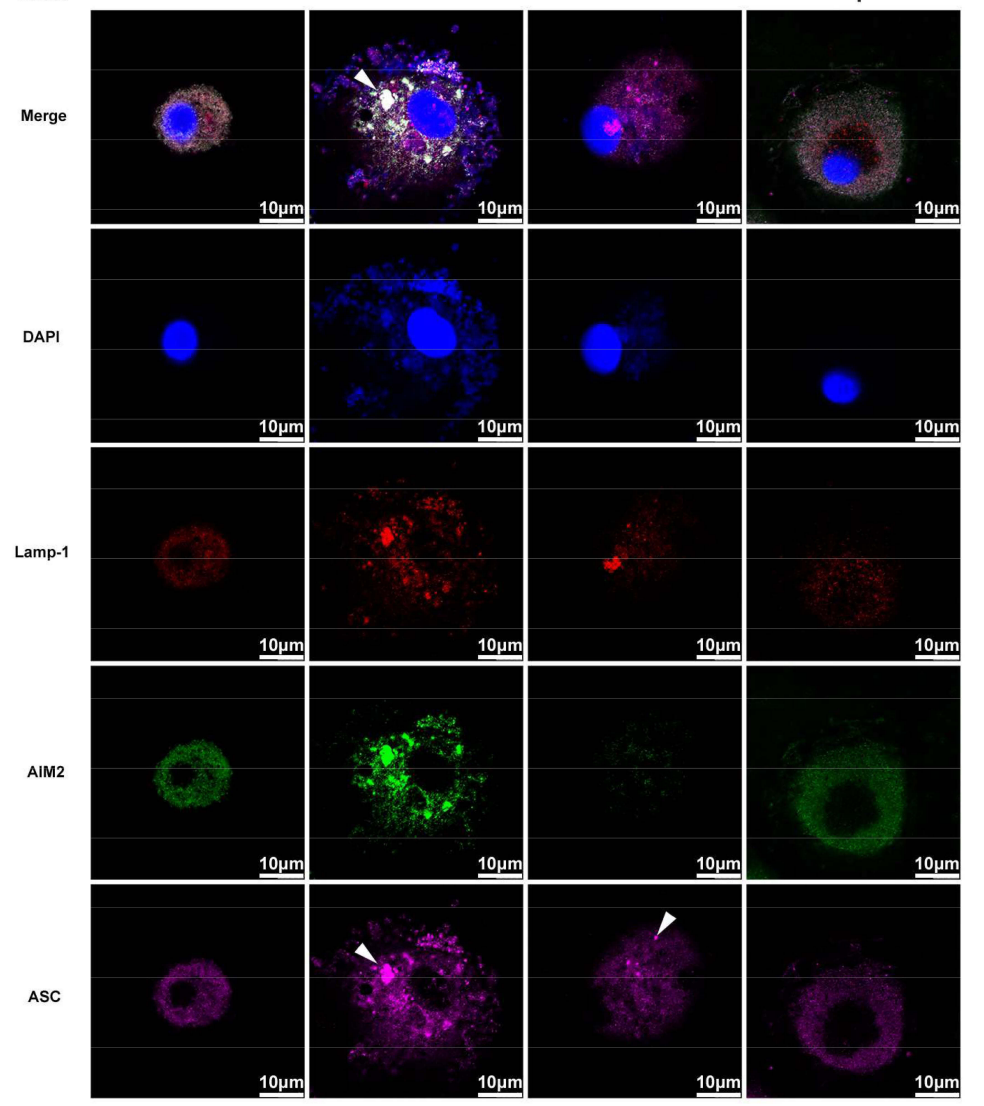

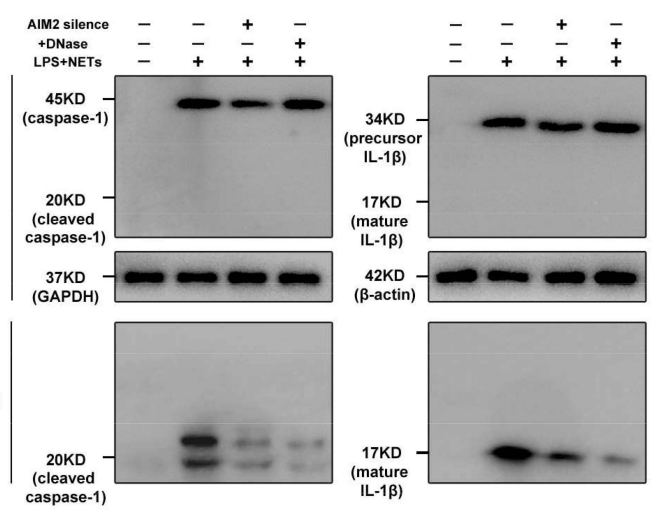

E

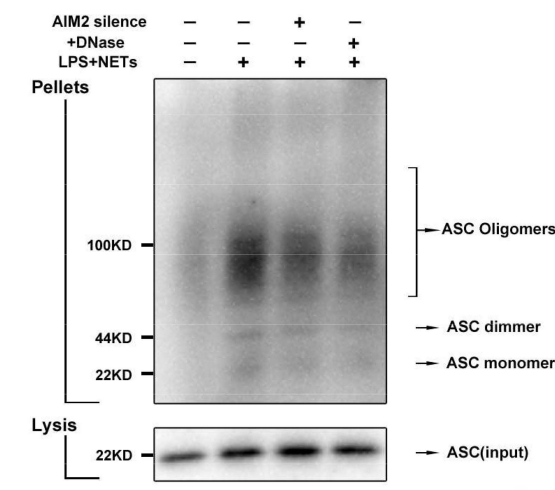

$\mathbf{F}$
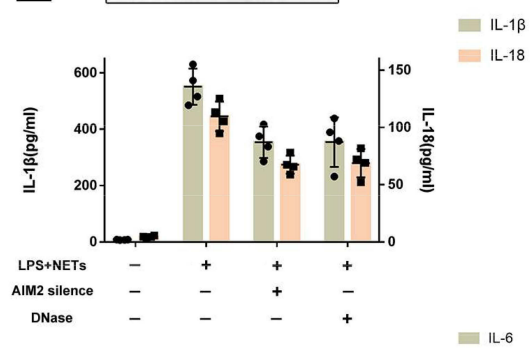

G

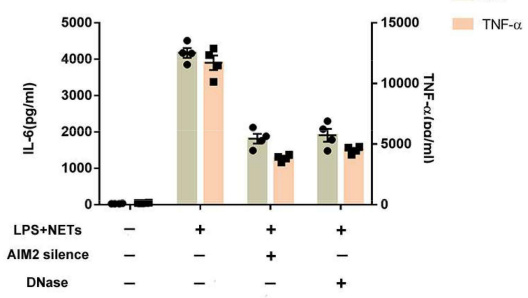

Figure 6 Silencing of the AIM2 gene or degradation of NET DNA alleviates alveolar macrophage pyroptosis. Silencing of the AIM2 gene or degradation of NET DNA decreased (A) LDH release, (B and $\mathbf{D})$ intracellular pyroptosome formation, (C) cleaved caspase-I and IL-I $\beta$ production and (E) ASC oligomerization. Administration of AIM2 siRNA and degradation of NET DNA also reduced (F) IL-I $\beta$, IL-I8, and (G) IL-6 and TNF-a levels in the supernatant. (**p<0.0I, $* * * * p<0.000$ I).

Abbreviations: NETs, neutrophil extracellular traps; AMs, alveolar macrophages; LPS, lipopolysaccharide; ASC, apoptosis-associated speck-like protein; IL-I $\beta$, interleukinI $\beta$; C/T, current NETs-DNA/total NETs-DNA; LDH, lactate dehydrogenase; AIM2, absent in melanoma 2.

Thus, we generated a murine model of LPS-induced ARDS and treated mice with agents to disrupt NETs or abolish alveolar macrophage pyroptosis to verify this hypothesis. In accordance with our previous results, the present study demonstrated that LPS challenge resulted in massive neutrophil and macrophage infiltration accompanied by an increase in NET content and caspase-1-dependent alveolar macrophage pyroptosis in BALF. When
NETs were degraded by DNase I or inhibited by BB-Clamidine, the NET content in BALF decreased, infiltration of neutrophils was not changed, and alveolar macrophage pyroptosis was attenuated. Additionally, abolishment of alveolar macrophage pyroptosis reduced both neutrophil influx and NET levels in BALF.

The observed reduction in alveolar macrophage pyroptosis could be attributed to a decreased level of neutrophil- 
derived NETs. Interestingly, we found that the trend in the reductions in neutrophil number/NET content reduction was surprisingly consistent. We also observed that the level of $\mathrm{KC}$, a neutrophil chemoattractant mainly secreted by macrophages, was declined in BALF when alveolar macrophage pyroptosis was suppressed. This rather intriguing finding might have resulted from the promotion of neutrophil infiltration by macrophage pyroptosis rather than NET generation. Thus, we conclude that the neutrophil-alveolar macrophage interaction in ARDS may have two effects: on the one hand, alveolar macrophage pyroptosis may lead to the recruitment of neutrophils following NET formation, and on the other hand, neutrophil-derived NETs may exacerbate alveolar macrophage pyroptosis. NET formation and macrophage pyroptosis could constitute a vicious cycle that contributes to aggravation of the cytokine storm and lung damage.

Our study further demonstrated the underlying mechanism and the role of NETs in alveolar macrophage pyroptosis. Consistent with our experimental observations, NETs can activate macrophages to release inflammatory cytokines, including IL-1 $\beta$, IL-6, and TNF-a, upon exposure to a pre-existing pathogen-associated molecular pattern (PAMP) (LPS) or damage-associated molecule pattern (DAMP) (uric acid). ${ }^{27,30,50,51}$ As a crucial inflammatory factor whose secretion is induced by caspase- 1 , IL- $1 \beta$ is a key proinflammatory cytokine involved in pyroptosis. Our findings suggested that pyroptosis was enhanced in alveolar macrophages costimulated with NETs and LPS to trigger AIM2 inflammasome activation. ALRs bind dsDNA (viral, bacterial, or even host) and act as cytosolic dsDNA sensors following the oligomerization of ASCs and inflammasome activation. This result may be explained by the fact that many NETs consist of a DNA backbone originating from neutrophil nuclear DNA and that AIM2, as a cytosolic DNA sensor, is capable of cleaving pro-caspase-1 in an ASC-dependent manner (AIM2 inflammasome formation). This is in accordance with previous finding that IL- $1 \beta$ is important for the response to AIM2 inflammasome activation. ${ }^{52-54}$

Macrophages phagocytose and degrade NETs without releasing inflammatory factors under physiological conditions. ${ }^{26}$ However, in our study, NET phagocytosis and degradation were affected by LPS exposure and cell type. One unexpected finding was that LPS priming of alveolar macrophages not only reduced the rate of phagocytosis of extracellular NETs but also slowed the rate of degradation of intracellular NETs. Consequently, NETs accumulated in LPS-primed alveolar macrophages. However, how the extracellular DNA backbone (NETs) is sensed by intracellular receptors (ALRs) has yet to be understood. Previous studies have shown that lysosomal dysfunction allows DNA to gain access to the cytosol and activate AIM2 in systemic lupus erythematosus and diabetes and that the AIM2 inflammasome is activated by pharmacological disruption of nuclear envelope integrity. ${ }^{55-58}$ LPS stimulation was also reported to induce ROS production, resulting in lysosomal destabilization. ${ }^{59}$ As in our study, NET DNA, which accumulated in the lysosomes of LPS-primed alveolar macrophages, was proven to bind with the AIM2 sensor to trigger the AIM2 inflammasome, possibly due to increased lysosomal membrane permeability. Degradation of NET DNA or silencing of the AIM2 gene can alleviate pyroptosis of alveolar macrophages costimulated with LPS and NETs, indirectly demonstrating that AIM2 inflammasome activation plays a role in alveolar macrophage pyroptosis.

Based on our results, we propose that neutrophilderived NETs and pyroptotic alveolar macrophages interact to mediate inflammation and lung injury after LPS challenge (Figure 7). In brief, LPS challenge results in alveolar damage and inflammatory cell infiltration. Neutrophils undergo NETosis to produce a large amount of NETs, which are directly cytotoxic to the epithelium and are engulfed by alveolar macrophages, leading to AIM2 inflammasome activation and caspase-1-dependent pyroptosis in LPS-priming alveolar macrophages followed by the release of a large amount of cytokines and more neutrophil infiltration, leading to ARDS through inflammatory storm development due to a vicious cycle.

In summary, we reveal some significant findings that could improve the understanding of lung inflammation during ARDS. First, accumulated intra-alveolar neutrophils that release NETs and alveolar macrophages are probably prone to pyroptosis in ARDS. There is a correlation between the release of NETs and pyroptosis. Second, in ARDS, NET formation exacerbates alveolar macrophage pyroptosis and lung injury, while alveolar macrophage pyroptosis enhances neutrophil chemotaxis in the alveolar cavity, which contributes to lung injury. We also found that NETs directly promote alveolar macrophage pyroptosis through NET DNA-mediated activation of the AIM2 inflammasome when combined with LPS stimulation. Consequently, NETs and the AIM2 sensor may be crucial therapeutic targets for the regulation of alveolar macrophage inflammasomemediated immunopathology in ARDS. 


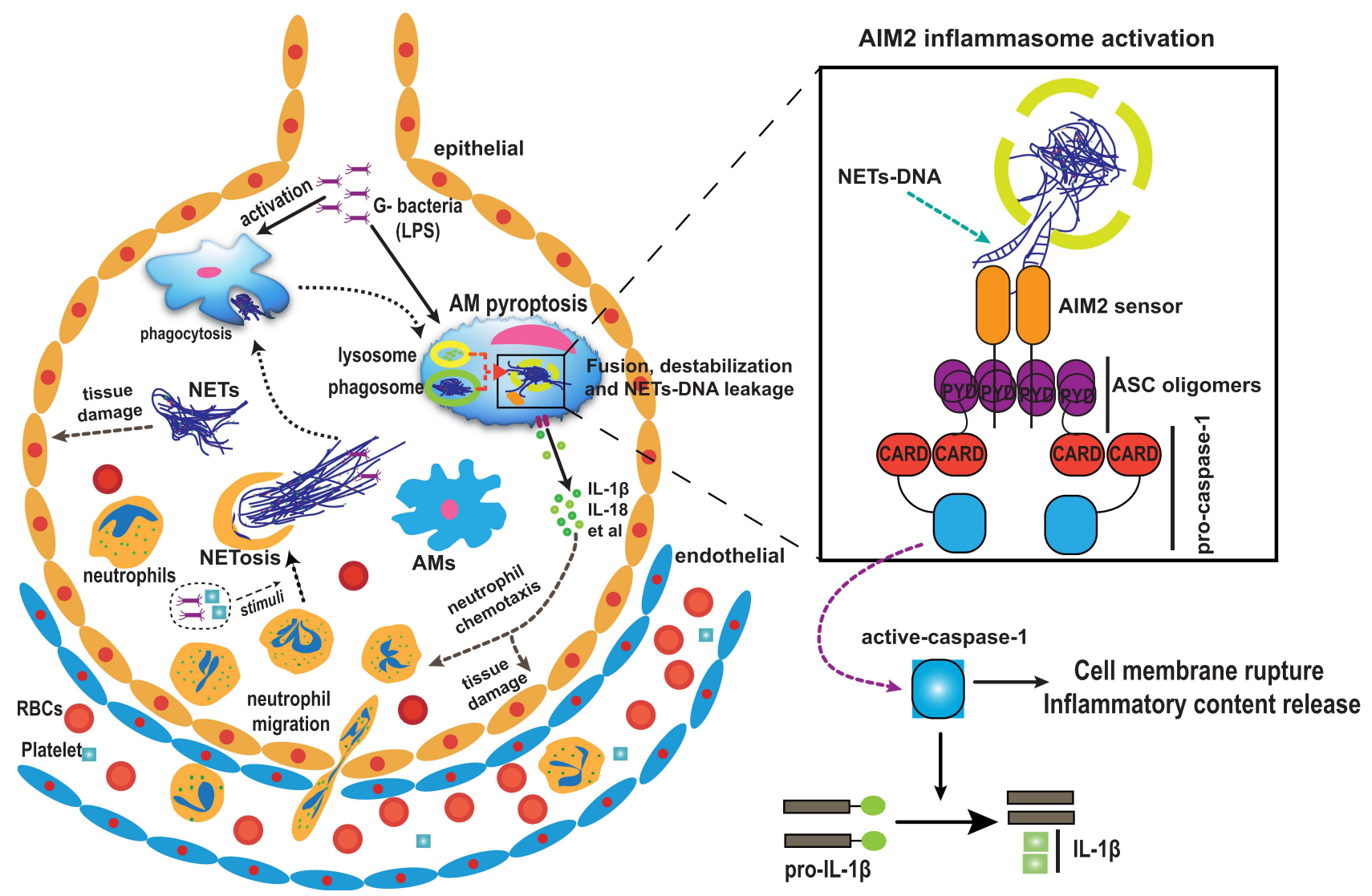

Figure 7 Hypothesized model of the mechanism by which NETs and pyroptotic alveolar macrophage interact to mediate of lung injury and inflammation in ARDS. It is proposed that NETs may originate from infiltrated neutrophils as a result of gram-negative bacteria and be engulfed by alveolar macrophages, causing AIM2 inflammasome activation and subsequent alveolar macrophage pyroptosis, which in turn results in the recruitment of more neutrophils and amplifies inflammation by promoting the production of cytokines that eventually contribute to the pathogenesis of ARDS.

Abbreviations: ARDS, acute respiratory distress syndrome; NETs, neutrophil extracellular traps; AIM2, absent in melanoma 2; RBC, red blood cell.

\section{Funding}

The study was supported by the National Natural Science Foundation of China (Grant No. 81770080, 82000089) National Key R\&D Program of China (No.2016YFC1304204), Project Program of National Clinical Research Center for Geriatric Disorders (Xiangya Hospital, Grant No.2020LNJJ05) and the Independent Explore Innovative Projects of Central South University (Grant No. 2017zzts208).

\section{Disclosure}

The authors report no conflicts of interest in this work.

\section{References}

1. Fan E, Brodie D, Slutsky AS. Acute respiratory distress syndrome: advances in diagnosis and treatment. JAMA. 2018;319(7):698-710. doi:10.1001/jama.2017.21907

2. Bellani G, Laffey JG, Pham T, et al. Epidemiology, patterns of care, and mortality for patients with acute respiratory distress syndrome in intensive care units in 50 countries. JAMA. 2016;315(8):788-800. doi:10.1001/jama.2016.0291
3. Alessandri F, Pugliese F, Ranieri VM. The role of rescue therapies in the treatment of severe ARDS. Respir Care. 2018;63(1):92-101. doi: $10.4187 /$ respcare 05752

4. Hussell T, Bell TJ. Alveolar macrophages: plasticity in a tissue-specific context. Nat Rev Immunol. 2014;14(2):81-93. doi: $10.1038 /$ nri3600

5. Wallach D, Kang TB, Dillon CP, Green DR. Programmed necrosis in inflammation: toward identification of the effector molecules. Science. 2016;352(6281):aaf2154. doi:10.1126/science.aaf2154

6. Fernandes-Alnemri T, Wu J, Yu JW, et al. The pyroptosome: a supramolecular assembly of ASC dimers mediating inflammatory cell death via caspase-1 activation. Cell Death Differ. 2007;14 (9):1590-1604. doi:10.1038/sj.cdd.4402194

7. Jorgensen I, Miao EA. Pyroptotic cell death defends against intracellular pathogens. Immunol Rev. 2015;265(1):130-142. doi:10.1111/imr.12287

8. Wu DD, Pan PH, Liu B, et al. Inhibition of alveolar macrophage pyroptosis reduces lipopolysaccharide-induced acute lung injury in mice. Chin Med J. 2015;128(19):2638-2645. doi:10.4103/03666999.166039

9. Wu D, Pan P, Su X, et al. Interferon regulatory factor-1 mediates alveolar macrophage pyroptosis during LPS-induced acute lung injury in mice. Shock. 2016;46(3):329-338. doi:10.1097/shk.00000 00000000595

10. Dolinay T, Kim YS, Howrylak J, et al. Inflammasome-regulated cytokines are critical mediators of acute lung injury. Am J Respir Crit Care Med. 2012;185(11):1225-1234. doi:10.1164/rccm.2012010003OC 
11. Jones HD, Crother TR, Gonzalez-Villalobos RA, et al. The NLRP3 inflammasome is required for the development of hypoxemia in LPS/ mechanical ventilation acute lung injury. Am J Respir Cell Mol Biol. 2014;50(2):270-280. doi:10.1165/rcmb.2013-0087OC

12. Kovarova M, Hesker PR, Jania L, et al. NLRP1-dependent pyroptosis leads to acute lung injury and morbidity in mice. J Immunol. 2012;189(4):2006-2016. doi:10.4049/jimmunol.1201065

13. Brodsky IE, Medzhitov R. Pyroptosis: macrophage suicide exposes hidden invaders. Curr Biol. 2011;21(2):R72-5. doi:10.1016/j.cub. 2010.12.008

14. Fink SL, Cookson BT. Pyroptosis and host cell death responses during Salmonella infection. Cell Microbiol. 2007;9(11):2562-2570. doi:10.1111/j.1462-5822.2007.01036.x

15. Zemans RL, Matthay MA. What drives neutrophils to the alveoli in ARDS? Thorax. 2017;72(1):1-3. doi:10.1136/thoraxjnl-2016-209170

16. Papayannopoulos V. Neutrophil extracellular traps in immunity and disease. Nat Rev Immunol. 2018;18(2):134-147. doi:10.1038/nri.20 17.105

17. Brinkmann V, Reichard U, Goosmann C, et al. Neutrophil extracellular traps kill bacteria. Science. 2004;303(5663):1532-1535. doi:10. 1126/science. 1092385

18. Huang H, Tohme S, Al-Khafaji AB, et al. Damage-associated molecular pattern-activated neutrophil extracellular trap exacerbates sterile inflammatory liver injury. Hepatology (Baltimore, Md). 2015;62 (2):600-614. doi:10.1002/hep.27841

19. Behnen M, Leschczyk C, Moller S, et al. Immobilized immune complexes induce neutrophil extracellular trap release by human neutrophil granulocytes via FcgammaRIIIB and Mac-1. J Immunol. 2014;193(4):1954-1965. doi:10.4049/jimmunol.1400478

20. Chen K, Nishi H, Travers R, et al. Endocytosis of soluble immune complexes leads to their clearance by FcgammaRIIIB but induces neutrophil extracellular traps via FcgammaRIIA in vivo. Blood. 2012;120(22):4421-4431. doi:10.1182/blood-2011-12-401133

21. Keshari RS, Jyoti A, Dubey M, et al. Cytokines induced neutrophil extracellular traps formation: implication for the inflammatory disease condition. PLoS One. 2012;7(10):e48111. doi:10.1371/journal. pone. 0048111

22. McDonald B, Davis RP, Kim SJ, et al. Platelets and neutrophil extracellular traps collaborate to promote intravascular coagulation during sepsis in mice. Blood. 2017;129(10):1357-1367. doi:10.1182/ blood-2016-09-741298

23. Narasaraju T, Yang E, Samy RP, et al. Excessive neutrophils and neutrophil extracellular traps contribute to acute lung injury of influenza pneumonitis. Am J Pathol. 2011;179(1):199-210. doi:10.1016/j. ajpath.2011.03.013

24. Li H, Zhou X, Tan H, et al. Neutrophil extracellular traps contribute to the pathogenesis of acid-aspiration-induced ALI/ARDS Oncotarget. 2018;9(2):1772-1784. doi:10.18632 oncotarget. 22744

25. Liu S, Su X, Pan P, et al. Neutrophil extracellular traps are indirectly triggered by lipopolysaccharide and contribute to acute lung injury. Sci Rep. 2016;6:37252. doi:10.1038/srep37252

26. Farrera C, Fadeel B. Macrophage clearance of neutrophil extracellular traps is a silent process. J Immunol. 2013;191(5):2647-2656. doi:10.4049/jimmunol.1300436

27. Warnatsch A, Ioannou M, Wang Q, Papayannopoulos V. Neutrophil extracellular traps license macrophages for cytokine production in atherosclerosis. Science. 2015;349(6245):316-320. doi:10.1126/ science.aaa8064

28. Riehl DR, Roewe J, Klebow S, et al. Neutrophil extracellular traps drive bleomycin-induced lung fibrosis by regulating TGF $\beta 1$-dependent interactions of platelets and macrophages. FASEB J. 2016;30(1_supplement):50.1. doi:10.1096/fasebj.30.1_supplement.50.1

29. Chen L, Zhao Y, Lai D, et al. Neutrophil extracellular traps promote macrophage pyroptosis in sepsis. Cell Death Dis. 2018;9(6):597. doi:10.1038/s41419-018-0538-5
30. Hu Z, Murakami T, Tamura H, et al. Neutrophil extracellular traps induce IL-1beta production by macrophages in combination with lipopolysaccharide. Int J Mol Med. 2017;39(3):549-558. doi:10. 3892/ijmm.2017.2870

31. Li H, Pan P, Su X, et al. Neutrophil extracellular traps are pathogenic in ventilator-induced lung injury and partially dependent on TLR4. Biomed Res Int. 2017;2017:8272504. doi:10.1155/2017/8272504

32. Knight JS, Subramanian V, O’Dell AA, et al. Peptidylarginine deiminase inhibition disrupts NET formation and protects against kidney, skin and vascular disease in lupus-prone MRL/lpr mice. Ann Rheum Dis. 2015;74(12):2199-2206. doi:10.1136/annrheumdis-2014-205365

33. Caudrillier A, Kessenbrock K, Gilliss BM, et al. Platelets induce neutrophil extracellular traps in transfusion-related acute lung injury. J Clin Invest. 2012;122(7):2661-2671. doi:10.1172/jci61303

34. Dicker AJ, Crichton ML, Pumphrey EG, et al. Neutrophil extracellular traps are associated with disease severity and microbiota diversity in patients with chronic obstructive pulmonary disease. J Allergy Clin Immunol. 2018;141(1):117-127. doi:10.1016/j.jaci.2017.04.022

35. Dai M, Pan P, Li H, et al. The antimicrobial cathelicidin peptide hlF (1-11) attenuates alveolar macrophage pyroptosis induced by Acinetobacter baumannii in vivo. Exp Cell Res. 2018;364 (1):95-103. doi:10.1016/j.yexcr.2018.01.035

36. Casals C, Arias-Diaz J, Valino F, et al. Surfactant strengthens the inhibitory effect of C-reactive protein on human lung macrophage cytokine release. Am J Physiol Lung Cell Mol Physiol. 2003;284(3): L466-72. doi:10.1152/ajplung.00325.2002

37. Gea-Sorli S, Guillamat R, Serrano-Mollar A, Closa D. Activation of lung macrophage subpopulations in experimental acute pancreatitis. J Pathol. 2011;223(3):417-424. doi:10.1002/path.2814

38. Czaikoski PG, Mota JM, Nascimento DC, et al. Neutrophil extracellular traps induce organ damage during experimental and clinical sepsis. PLoS One. 2016;11(2):e0148142. doi:10.1371/journal.pone.0148142

39. Lugrin J, Martinon F. Detection of ASC oligomerization by western blotting. Bio-Protocol. 2017;7(10):e2292. doi:10.21769/BioProtoc. 2292

40. Ryu JC, Kim MJ, Kwon Y, et al. Neutrophil pyroptosis mediates pathology of $\mathrm{P}$. aeruginosa lung infection in the absence of the NADPH oxidase NOX2. Article. Mucosal Immunol. 2016;10:757. doi: $10.1038 / \mathrm{mi} .2016 .73$

41. Chen KW, Gross CJ, Sotomayor FV, et al. The neutrophil NLRC4 inflammasome selectively promotes IL-1beta maturation without pyroptosis during acute Salmonella challenge. Cell Rep. 2014;8 (2):570-582. doi:10.1016/j.celrep.2014.06.028

42. Máca J, Jor O, Holub M, et al. Past and present ARDS mortality rates: a Systematic Review. Respir Care. 2017;62(1):113-122. doi: $10.4187 /$ respcare. 04716

43. Kallet R, Pirracchio R, Lipnick MS, Sinha P, Gomez A, Matthay MA. Mortality rate in ARDS is higher in those meeting NIH ARDSNet exclusion criteria. Respir Care. 2018;63(Suppl 10):3017278.

44. Soehnlein O, Lindbom L. Phagocyte partnership during the onset and resolution of inflammation. Nat Rev Immunol. 2010;10(6):427-439. doi:10.1038/nri2779

45. Yang W, Tao Y, Wu Y, et al. Neutrophils promote the development of reparative macrophages mediated by ROS to orchestrate liver repair. Nat Commun. 2019;10(1):1076. doi:10.1038/s41467-019-09046-8

46. Prame Kumar K, Nicholls AJ, Wong CHY. Partners in crime: neutrophils and monocytes/macrophages in inflammation and disease. Cell Tissue Res. 2018;371(3):551-565. doi:10.1007/s00441-017-2753-2

47. Sadatomo A, Inoue $\mathrm{Y}$, Ito $\mathrm{H}$, et al. Interaction of neutrophils with macrophages promotes IL-1 $\beta$ maturation and contributes to hepatic ischemia-reperfusion injury. J Immunol. 2017;199(9):3306-3315. doi:10.4049/jimmunol.1700717

48. Aktas G, Duman T, Atak B, et al. Irritable bowel syndrome is associated with novel inflammatory markers derived from hemogram parameters. journal article. Family Med Prim Care Rev. 2020;22 (2):107-110. doi:10.5114/fmpcr.2020.95311 
49. Liu S, Yue Y, Pan P, et al. IRF-1 intervention in the classical ROS-dependent release of NETs during LPS-induced acute lung injury in mice. Inflammation. 2019;42(1):387-403. doi:10.1007/ s10753-018-0903-7

50. Lazzaretto B, Fadeel B. Intra- and extracellular degradation of neutrophil extracellular traps by macrophages and dendritic cells. J Immunol. 2019;203(8):2276-2290. doi:10.4049/jimmunol.1800159

51. Josefs T, Barrett TJ, Brown EJ, et al. Neutrophil extracellular traps promote macrophage inflammation and impair atherosclerosis resolution in diabetic mice. JCI Insight. 2020;5(7):e134796. doi:10.1172/ jci.insight. 134796

52. Trachalaki A, Tsitoura E, Mastrodimou S, et al. Enhanced IL-1 $\beta$ release following NLRP3 and AIM2 inflammasome stimulation is linked to mtROS in airway macrophages in pulmonary fibrosis. Original Research. Front Immunol. 2021;12:2182. doi:10.3389/ fimmu.2021.661811

53. Fidler TP, Xue C, Yalcinkaya M, et al. The AIM2 inflammasome exacerbates atherosclerosis in clonal haematopoiesis. Nature. 2021;592(7853):296-301. doi:10.1038/s41586-021-03341-5

54. Roth S, Cao J, Singh V, et al. Post-injury immunosuppression and secondary infections are caused by an AIM2 inflammasome-driven signaling cascade. Immunity. 2021;54(4):648-659.e8. doi:10.1016/j. immuni.2021.02.004
55. Bai Y, Tong Y, Liu Y, Hu H. Self-dsDNA in the pathogenesis of systemic lupus erythematosus. Clin Exp Immunol. 2018;191(1):1-10. doi:10.1111/cei.13041

56. Sharma BR, Karki R, Kanneganti T-D. Role of AIM2 inflammasome in inflammatory diseases, cancer and infection. Eur J Immunol. 2019;49(11):1998-2011. doi:10.1002/eji.201848070

57. Bae JH, Jo S, Kim SJ, et al. Circulating cell-free mtDNA contributes to AIM2 inflammasome-mediated chronic inflammation in patients with Type 2 diabetes. Cells. 2019;8(4):328. doi:10.3390/ cells 8040328

58. Houthaeve G, Xiong R, Robijns J, et al. Targeted perturbation of nuclear envelope integrity with vapor nanobubble-mediated photoporation. ACS Nano. 2018;12(8):7791-7802. doi:10.1021/ acsnano. $8 \mathrm{~b} 01860$

59. Luo Y, Xiong B, Liu H, et al. Koumine suppresses IL-1 $\beta$ secretion and attenuates inflammation associated with blocking ROS/NF- $\mathrm{BB} /$ NLRP3 axis in macrophages. Front Pharmacol. 2021;11:622074. doi:10.3389/fphar.2020.622074
Journal of Inflammation Research

\section{Publish your work in this journal}

The Journal of Inflammation Research is an international, peerreviewed open-access journal that welcomes laboratory and clinical findings on the molecular basis, cell biology and pharmacology of inflammation including original research, reviews, symposium reports, hypothesis formation and commentaries on: acute/chronic inflammation; mediators of inflammation; cellular processes; molecular
Dovepress

mechanisms; pharmacology and novel anti-inflammatory drugs; clinical conditions involving inflammation. The manuscript management system is completely online and includes a very quick and fair peerreview system. Visit http://www.dovepress.com/testimonials.php to read real quotes from published authors. 